\title{
ON QUANTUM COHOMOLOGY RINGS OF PARTIAL FLAG VARIETIES
}

\author{
Ionuţ Ciocan-Fontanine \\ Institut Mittag-Leffler
}

February 9, 1997

\section{INTRODUCTION}

The main goal of this paper is to give a unified description for the structure of the small quantum cohomology rings for all homogeneous spaces of $S L_{n}(\mathbb{C})$.

The quantum cohomology ring of a smooth projective variety, or, more generally of a symplectic manifold, has been introduced by physicists in the study of topological field theories $([\mathrm{V}],[\mathrm{W}])$. In the past few years, the highly non-trivial task of giving a rigorous mathematical treatment for the theory of quantum cohomology has been accomplished, both in the realm of algebraic and symplectic geometry. In various degrees of generality, this can be found in $[\mathrm{B}],[\mathrm{BM}],[\mathrm{KM}]$, [Kon] [LT1], $[\mathrm{LT} 2]$, [MS], [RT], as well as the surveys [FP] and [T]. Roughly speaking, the quantum cohomology ring of a variety $X$ is a deformation of the usual cohomology ring, with parameter space given by $H^{*}(X)$. The multiplicative structure of quantum cohomology encodes the enumerative geometry of rational curves on $X$

If one restricts the parameter space to $H^{1,1}(X)$, one gets the small quantum cohomology ring (terminology taken from $[\mathrm{FP}]$ ). This ring, in the case of partial flag varieties is the object of the present paper. In order to state our main results, we will first describe briefly the "classical" side of the story.

Let $P$ be a parabolic subgroup of $S L_{n}(\mathbb{C})$. We will interpret the homogeneous space $F:=S L_{n}(\mathbb{C}) / P$ as the complex projective variety parametrizing flags of quotients of $\mathbb{C}^{n}$ of given ranks, say $n_{k}>\cdots>n_{1}$.

By a classical result of C. Ehresmann ([E]), the integral cohomolgy of $F$ can be described geometrically as the free abelian group generated by the Schubert classes. These are the (Poincaré duals of) fundamental classes of certain subvarieties $\Omega_{w} \subset$ $F$, one for each element of the subset $S:=S\left(n_{1}, \ldots, n_{k}\right)$ of the symmetric group $S_{n}$, consisting of permutations $w$ such that if $w(i)>w(i+1)$, then $i \in\left\{n_{1}, \ldots, n_{k}\right\}$.

A description of the multiplicative structure is provided by yet another classical theorem, due to A. Borel ([Bor]), which gives a presentation for $H^{*}(F, \mathbb{Z})$. Specifically, let $\sigma_{1}^{1}, \ldots, \sigma_{n_{1}}^{1}, \sigma_{1}^{2}, \ldots, \sigma_{n_{2}-n_{1}}^{2}, \ldots, \sigma_{1}^{k+1}, \ldots, \sigma_{n-n_{k}}^{k+1}$ be $n$ independent variables. Define $A_{n}$ to be the block diagonal matrix $\operatorname{diag}\left(D_{1}, D_{2}, \ldots, D_{k+1}\right)$, where

$$
D_{j}:=\left(\begin{array}{ccccc}
\sigma_{1}^{j} & \sigma_{2}^{j} & \ldots & \sigma_{n_{j}-n_{j-1}-1}^{j} & \sigma_{n_{j}-n_{j-1}}^{j} \\
-1 & 0 & \ldots & 0 & 0 \\
\vdots & \vdots & \ldots & \vdots & \vdots
\end{array}\right)
$$


Borel's result then states that there is a canonical isomorphism

$$
\mathbb{Z}\left[\sigma_{1}^{1}, \ldots, \sigma_{n_{1}}^{1}, \sigma_{1}^{2}, \ldots, \sigma_{n_{2}-n_{1}}^{2}, \ldots, \sigma_{1}^{k+1}, \ldots, \sigma_{n-n_{k}}^{k+1}\right] /\left(g_{1}, g_{2}, \ldots, g_{n}\right) \cong H^{*}(F),
$$

where the $g_{j}$ 's are the coefficients of the characteristic polynomial of the matrix $A_{n}$.

The natural problem arising from the above descriptions is to look for polynomial representatives for the Schubert classes. The first case in which this problem has been solved is when $F$ is a Grassmannian, and goes back to G. Giambelli.

The general case was obtained independently by I. N. Bernstein, I. M. Gelfand, and S. I. Gelfand ([BGG]), and M. Demazure ([D]). In fact, it should be noted that it suffices to solve the above problem for the complete flag variety $F_{n}=S L_{n}(\mathbb{C}) / B$. The point is that the map

$$
H^{*}(F, \mathbb{Z}) \longrightarrow H^{*}\left(F_{n}, \mathbb{Z}\right)
$$

induced by flat pull-back via the natural projection $F_{n} \rightarrow F$ is an embedding. To be more precise, the Borel description for the cohomology of the complete flag variety is

$$
\mathbb{Z}\left[x_{1}, x_{2}, \ldots, x_{n}\right] /\left(e_{1}, \ldots, e_{n}\right) \cong H^{*}\left(F_{n}, \mathbb{Z}\right),
$$

where $e_{j}$ is the $j^{\text {th }}$ elementary symmetric polynomial in $x_{1}, \ldots, x_{n}$. A particulary nice set of representatives for the Schubert classes in this case are the $S c h u$ bert polynomials $\mathfrak{S}_{w}\left(x_{1}, \ldots, x_{n}\right)$ of Lascoux and Schützenberger ([LS1], [LS2]). If we interpret each $\sigma_{i}^{j}$ as the $i^{\text {th }}$ elementary symmetric polynomial in variables $x_{n_{j-1}+1}, \ldots, x_{n_{j}}$, then the image of $H^{*}(F, \mathbb{Z})$ by the map $(0.2)$ is the subring of polynomials which are symmetric in variables in each of the groups

$$
\underbrace{x_{1} \ldots, x_{n_{1}}}, \underbrace{x_{n_{1}+1}, \ldots, x_{n_{2}}}, \ldots, \underbrace{x_{n_{k}+1}, \ldots, x_{n}} .
$$

If $w \in S$, then $\mathfrak{S}_{w}$ satisfies the above symmetry, hence it determines a polynomial $P_{w}$ in the $\sigma$ variables, which represents $\left[\Omega_{w}\right]$ in $H^{*}(F, \mathbb{Z})$. We will call the $P_{w}(\sigma)^{\prime}$ 's the Giambelli polynomials associated to $F$.

Among the Schubert varieties, there are the so-called special Schubert varieties, which are geometric realizations of the Chern classes of the universal quotient bundles on $F$. They correspond to the cyclic permutations $\alpha_{i, j}:=s_{n_{j}-i+1} \cdot \ldots \cdot s_{n_{j}}$, $1 \leq j \leq k, 1 \leq i \leq n_{j}$, where $s_{m}:=(m, m+1)$ is the simple transposition interchanging $m$ and $m+1$. Again, when $F$ is a Grassmannian, there is a classical formula, due to M. Pieri, expressing the product $\left[\Omega_{\alpha_{i, 1}}\right] \cdot\left[\Omega_{w}\right]$ in the basis of Schubert classes. Its generalization to the case of the complete flag variety, hence, by the above discussion, to any partial flag variety as well, was first stated by Lascoux and Schützenberger ([LS1]), and was given a geometric proof only recently by F. Sottile $([\mathrm{S}])$.

In analogy to the case of Grassmannians, we will refer to the Giambelli and Pieri-type formulae as the classical Schubert Calculus on $F$.

The small quantum cohomology ring of $F$, denoted by $Q H^{*}(F)$, is defined as the $\mathbb{Z}\left[q_{1}, \ldots, q_{k}\right]$-module $H^{*}(F, \mathbb{Z}) \otimes_{\mathbb{Z}} \mathbb{Z}\left[q_{1}, \ldots, q_{k}\right]$, where $q_{1}, \ldots, q_{k}$ are formal variables, with a new multiplication, which we denote by $*$, obtained essentially by replac- 
invariants of $F$. A presentation of $Q H^{*}(F)$ has been given independently by A. Astashkevich and V. Sadov ([AS]), and B. Kim ([Kim1]), with the proof completed in [Kim2] (the "extreme" cases of Grassmannians and complete flags were established slightly earlier in [ST], and [C-F1] and [GK] respectively). Their result is as follows. Let $B_{n}=\left(b_{l m}\right)_{1 \leq l, m \leq n}$ be the matrix with entries

$$
b_{l m}= \begin{cases}(-1)^{n_{j+1}-n_{j}+1} q_{j}, & \text { if } l=n_{j-1}+1, m=n_{j+1}, 1 \leq j \leq k \\ -1, & \text { if } l=n_{j}+1, m=n_{j}, 1 \leq j \leq k-1 \\ 0, & \text { otherwise }\end{cases}
$$

Then there exists a canonical isomorphism

$$
\mathbb{Z}\left[\sigma_{1}^{1}, \ldots, \sigma_{n_{1}}^{1}, \ldots, \sigma_{1}^{k+1}, \ldots, \sigma_{n-n_{k}}^{k+1}\right]\left[q_{1} \ldots, q_{k}\right] /\left(G_{1}, \ldots, G_{n}\right) \cong Q H^{*}(F),
$$

where $G_{1}, \ldots, G_{n}$ are the coefficients of the characteristic polynomial of the deformed matrix $A_{n}^{q}:=A_{n}+B_{n}$.

From the point of view of enumerative geometry, one is interested in computing the Gromov-Witten invariants of $F$, and the description (0.3) is not too helpful, unless one has quantum versions of the Giambelli and Pieri formulas. In other words, one is interested in developing a Quantum Schubert Calculus. The first such formulas, in the case $F$ is a Grassmannian, were discovered by A. Bertram, whose paper $[\mathrm{Be}]$ pioneered the subject. Later on, his approach was extended to the case of complete flags, to obtain the quantum Giambelli formula for the special Schubert classes (see [C-F1], [C-F2]). Using this, the Quantum Schubert polynomials were constructed with algebro-combinatorial methods by S. Fomin, S. Gelfand and A. Postnikov ([FGP]), giving therefore the full quantum Giambelli formula for the variety of complete flags. They have also given a special case of the Quantum Pieri formula, namely the Quantum Monk formula, which corresponds to multiplying by the first Chern class of one of the tautological bundles.

As opposed to the situation of the classical cohomology, the quantum story for a partial flag variety is far from being determined by the one for the complete flags, the reason being that the quantum cohomology lacks the functoriality enjoyed by the usual one. The main results of this paper are unified quantum versions of the Giambelli and Pieri formulas, which hold for any $F$. These formulas specialize to the ones described above when $F$ is either a Grassmannian, or the complete flag variety. In order to state them, we introduce first some notation.

Let

$$
1 \leq h_{1}<\cdots<h_{m} \leq l_{m}<\cdots<l_{1} \leq k
$$

be integers. We denote by $\mathbf{h}$, respectively $\mathbf{l}$, the collections $h_{1}, \ldots, h_{m}$ and $l_{1}, \ldots, l_{m}$. Let

$$
\begin{gathered}
\gamma_{\mathbf{h l}}:=\gamma_{h_{m}, l_{m}} \cdot \gamma_{h_{m-1}, l_{m-1}} \cdot \ldots \cdot \gamma_{h_{1}, l_{1}}, \\
\delta_{\mathbf{h l}}:=\delta_{h_{1}, l_{1}} \cdot \delta_{h_{2}, l_{2}} \cdot \ldots \cdot \delta_{h_{m}, l_{m}}
\end{gathered}
$$

where $\gamma_{h, l}$ and $\delta_{h, l}$ denote the cyclic permutations $s_{n_{h}} \cdot \ldots \cdot s_{n_{l+1}-1}$ and $s_{n_{l}-1} \cdot \ldots$. $s_{n_{h-1}+1}$ respectively, for any integers $h, l$ satisfying $1 \leq h \leq l \leq k$.

Denote by $q_{\mathbf{h l}}$ the monomial

$$
q_{\mathbf{h l}}:=q_{h_{1}} \ldots q_{h_{2}-1} q_{h_{2}}^{2} \ldots q_{h_{3}-1}^{2} \ldots q_{h_{m}}^{m} \ldots q_{l_{m}}^{m} q_{l_{m}-1}^{m-1} \ldots q_{l_{m-1}}^{m-1} \ldots q_{l_{2}-1} \ldots q_{l_{1}}
$$


For each $1 \leq j \leq k$ and $1 \leq i \leq n_{j}$, let $\alpha_{i, j}=s_{n_{j}-i+1} \cdot \ldots \cdot s_{n_{j}}$. For $1 \leq a<b \leq n$ denote by $t_{a b}$ the transposition interchanging $a$ and $b$. If $w, w^{\prime} \in S$, write $w \stackrel{\alpha_{i, j}}{\longrightarrow} w^{\prime}$ if there exist integers $a_{1}, b_{1}, \ldots, a_{i}, b_{i}$, such that

(1) $a_{r} \leq n_{j}<b_{r}$, for $1 \leq r \leq i$ and $w^{\prime}=w \cdot t_{a_{1} b_{1}} \cdot \ldots \cdot t_{a_{i} b_{i}}$;

(2) $\ell\left(w \cdot t_{a_{1} b_{1}} \cdot \ldots \cdot t_{a_{r} b_{r}}\right)=\ell(w)+r, 1 \leq r \leq i$;

(3) the integers $a_{1}, \ldots, a_{i}$ are distinct.

Our first main theorem is the

Quantum Pieri formula. For every $1 \leq j \leq k, 1 \leq i \leq n_{j}$ and $w \in S$,

$$
\left[\Omega_{\alpha_{i, j}}\right] *\left[\Omega_{w}\right]=\sum_{\substack{\alpha_{i, j} \\ w}}\left[\Omega_{w^{\prime}}\right]+\sum_{\mathbf{h}, \mathbf{l}} q_{\mathbf{h l}}\left(\sum_{w^{\prime \prime}}\left[\Omega_{w^{\prime \prime} \cdot \delta_{\mathbf{h l}}}\right]\right),
$$

where the second sum is over all collections $\mathbf{h}, \mathbf{l}$ such that $m \leq i, h_{m} \leq j \leq l_{m}$, and

$$
\ell\left(w \cdot \gamma_{\mathbf{h} \mathbf{l}}\right)=\ell(w)-\sum_{c=1}^{m}\left(n_{l_{c}+1}-n_{h_{c}}\right)
$$

while the last sum is over all permutations $w^{\prime \prime} \in S_{n}$ satisfying $w \cdot \gamma_{\mathbf{h l}} \stackrel{\tilde{\alpha}_{i, j}}{\longrightarrow} w^{\prime \prime}$, with $\tilde{\alpha}_{i, j}=\alpha_{i, j} \cdot s_{n_{j}} \cdot s_{n_{j}-1} \cdot \ldots \cdot s_{n_{j}-m+1}$, and

$$
\ell\left(w^{\prime \prime} \cdot \delta_{\mathbf{h l}}\right)=\ell\left(w^{\prime \prime}\right)-m-\sum_{c=1}^{m}\left(n_{l_{c}}-n_{h_{c}-1}\right)
$$

For each $1 \leq j \leq k, 1 \leq i \leq n_{j}$, let $g_{i}^{j}=g_{i}^{j}(\sigma)$ be the polynomial representing the $i^{\text {th }}$ Chern class of the $j^{\text {th }}$ universal quotient bundle on $F$. Alternatively, for each $j$, the polynomials $g_{i}^{j}, 1 \leq i \leq n_{j}$ are the coefficients of the characteristic polynomial $\operatorname{det}\left(A_{n_{j}}+\lambda I\right)$, where $A_{n_{j}}$ is the upper left $n_{j} \times n_{j}$ submatrix of the matrix $A_{n}$.

Define now polynomials $G_{i}^{j}=G_{i}^{j}(\sigma, q), 1 \leq j \leq k 1 \leq i \leq n_{j}$ in exactly the same way as above, but using the Astashkevich-Sadov-Kim matrix $A_{n}^{q}$ instead of $A_{n}$

For a partition $\Lambda_{j}:=\left(\lambda_{j, 1}, \ldots, \lambda_{j, n_{j+1}-n_{j}}\right)$ with (at most) $n_{j+1}-n_{j}$ parts and such that each part $\lambda_{j, m}$ is at most $n_{j}$, set

$$
g_{\Lambda_{j}}^{(j)}:=g_{\lambda_{j, 1}}^{j} g_{\lambda_{j, 2}}^{j} \ldots g_{\lambda_{j, n_{j+1}-n_{j}}^{j}}^{j} .
$$

Define "standard elementary monomials" $g_{\Lambda}:=g_{\Lambda_{1} \Lambda_{2} \ldots \Lambda_{k}} \in \mathbb{Z}[\sigma]$ by

$$
g_{\Lambda}:=g_{\Lambda_{1}}^{(1)} g_{\Lambda_{2}}^{(2)} \ldots g_{\Lambda_{k}}^{(k)}
$$

Similarly, the standard quantum elementary monomial $G_{\Lambda}$ is the polynomial in $\mathbb{Z}[\sigma, q]$ obtained by replacing in $g_{\Lambda}$ each factor $g_{\lambda}^{j}$ by the corresponding $G_{\lambda}^{j}$. It is easy to see that that each Giambelli polynomial can be written uniquely as a linear combination $P_{w}=\sum_{\Lambda} a_{\Lambda}(w) g_{\Lambda}$, with $a_{\Lambda}(w)$ integers.

Following [FGP], we define the quantum Giambelli polynomial $P_{w}^{q}(\sigma, q)$ by

$$
P_{w}^{q}(\sigma, q)=\sum_{\Lambda} a_{\Lambda}(w) G_{\Lambda}
$$


Quantum Giambelli formula. $\left[\Omega_{w}\right]=P_{w}^{q}(\sigma, q)$ in $Q H^{*}(F)$, for all $w \in S$.

We describe now briefly the way our proofs go. For the proof of Quantum Pieri we use the construction of 3-point Gromov-Witten invariants by means of hyperquot schemes. By a certain degeneration technique, the computation of some of these invariants is reduced to evaluating intersection numbers on $F$ itself. All the ideas involved here appear already in the geometric proof of the quantum Monk formula given in $[\mathrm{C}-\mathrm{F} 2]$.

Since the above definition of quantum Giambelli polynomials is the straightforward extension of the one given in [FGP] for the complete flag variety, the very nice and simple proof given there for the quantum Giambelli formula will work in the partial flag case too, once all the crucial ingredients are in place. More precisely, what needs to be shown is first that the formula holds for the special Schubert classes, and secondly that the quantum Giambelli polynomials defined above are orthogonal with respect to a naturally defined inner product on the quotient $\mathbb{Z}[\sigma, q] / I_{q}$ (see $(0.3))$.

The first result follows rather easily from the quantum Pieri formula (cf. also [C-F2]). As a byproduct, we also get an independent proof for the AstashkevichSadov-Kim theorem (0.3).

The proof of the orthogonality property given in $[\mathrm{FGP}]$ is combinatorial, and is the technical heart of their paper. Rather than trying to extend their method to the case of partial flags, we provide here a geometric proof. Namely, by using the fact that the quantum Giambelli formula holds for the special Schubert classes, we reduce the orthogonality to a statement about vanishing of certain Gromov-Witten invariants. The later is then shown by a degeneration argument similar to the one in the proof of quantum Pieri.

The paper is divided into two main parts. The first three sections contain a quick review of the results about the classical and quantum cohomology rings that we will need, and the proof of the quantum Giambelli formula, assuming that Quantum Pieri and orthogonality hold. The last three sections study the geometry of hyperquot schemes, from which we deduce the proofs of Quantum Pieri and orthogonality.

\section{Acknowledgements.}

I have learned the subject from Aaron Bertram, and many of the ideas that are used in this paper originate in his work on quantum cohomology of Grassmannians. I am also indebted to William Fulton, and Bumsig Kim, for useful discussions during the preparation of the paper. Financial support, via a postdoctoral fellowship, as well as a stimulating atmosphere for research, has been provided by the MittagLeffler Institute.

\section{The Classical Cohomology Ring}

\subsection{Schubert varieties.}

Let $0=n_{0}<n_{1}<n_{2}<\cdots<n_{k}<n_{k+1}=n$ be integers. Let $V$ be a complex $n$-dimensional vector space. The data $k, n_{j}, j=0, \ldots, k+1$, and $V$ will be fixed for the rest of the paper. Define $F:=F\left(n_{1}, \ldots, n_{k}, V\right)$ to be the variety parametrizing 
projective variety, of dimension $f:=\sum_{j=1}^{k}\left(n-n_{j}\right)\left(n_{j}-n_{j-1}\right)$. It comes with a tautological sequence of quotient bundles

$$
V_{F}:=V \otimes \mathcal{O}_{F} \rightarrow Q_{k} \rightarrow Q_{k-1} \rightarrow \cdots \rightarrow Q_{1},
$$

with $\operatorname{rank}\left(Q_{j}\right)=n_{j}$.

Let $S_{n}$ be the symmetric group on $n$ letters and let $S:=S\left(n_{1}, \ldots, n_{k}\right) \subset S_{n}$ be the subset consisting of permutations $w$ satisfying the condition: if $w(q)>w(q+1)$, then $q \in\left\{n_{1}, \ldots, n_{k}\right\}$. In other words, when regarded as a function $[1, n] \rightarrow[1, n]$, $w$ is increasing on each of the intervals $\left[1, n_{1}\right],\left[n_{1}+1, n_{2}\right], \ldots\left[n_{k}, n_{k+1}\right]$. The rank function of a permutation $w \in S_{n}$ is defined by

$$
r_{w}(q, p)=\operatorname{card}\{i \mid i \leq q, w(i) \leq p\}, 1 \leq q, p \leq n .
$$

Fix a complete flag of subspaces $V_{1} \subset V_{2} \subset \cdots \subset V_{n-1} \subset V_{n}=V$. For $w \in S$, the corresponding Schubert variety is defined by

$$
\Omega_{w}:=\left\{x \in F \mid \operatorname{rank}_{x}\left(V_{p} \otimes \mathcal{O} \rightarrow Q_{q}\right) \leq r_{w}(q, p), q \in\left\{n_{1}, \ldots, n_{k}\right\}, 1 \leq p \leq n\right\} .
$$

$\Omega_{w}$ is an irreducible subvariety in $F$, of (complex) codimension equal to the length $\ell(w)$ of the permutation $w$.

Throughout the paper $H^{*}(F)$ will denote the integral cohomolgy of $F$. The following two theorems are classical results of C. Ehresmann ([E], see also $[\mathrm{F} 2$, Example 14.7.16]).

Theorem 1.1 (Basis). $\left\{\left[\Omega_{w}\right]\right\}_{w \in S}$ freely generate $H^{*}(F)$ over $\mathbb{Z}$.

Theorem 1.2 (Duality). For every $w \in S$ there exists an unique permutation $\check{w} \in S$ such that

$$
\int_{F}\left[\Omega_{w}\right] \cup\left[\Omega_{v}\right]=\left\{\begin{array}{ll}
1, & \text { if } v=\check{w} \\
0, & \text { otherwise }
\end{array} .\right.
$$

\subsection{A presentation of $H^{*}(F)$.}

Consider on $F$ the vector bundles

$$
L_{j}:=\operatorname{ker}\left(Q_{j} \rightarrow Q_{j-1}\right),
$$

and let $\sigma_{i}^{j}:=c_{i}\left(L_{j}\right), 1 \leq i \leq n_{j}-n_{j-1}, 1 \leq j \leq k+1$. Let $x_{1}, \ldots, x_{n}$ be independent variables. For all $0 \leq i \leq m \leq n$, let $e_{i}^{m}$ denote the $i^{\text {th }}$ elementary symmetric function in the variables $x_{1} \ldots, x_{m}$. We regard the variables in each of the groups

$$
\underbrace{x_{1} \ldots, x_{n_{1}}}, \underbrace{x_{n_{1}+1}, \ldots, x_{n_{2}}}, \ldots, \underbrace{x_{n_{k}+1}, \ldots, x_{n}}
$$

as the Chern roots of the bundles $Q_{1}, L_{2}, \ldots, L_{k+1}$ respectively. For each $1 \leq j \leq$ $k+1$, the polynomials $e_{i}^{n_{j}}$ can be written as polynomials $g_{i}^{j}=g_{i}^{j}\left(\sigma_{1}^{1}, \ldots, \sigma_{n_{k+1}-n_{k}}^{k}\right)$ in the Chern classes of these bundles. $g_{i}^{j}$ has weighted degree $i$, where each $\sigma_{m}^{*}$ is assigned degree $m$. In particular, we have polynomials $g_{i}^{k+1}$ for $1 \leq i \leq n$.

We will denote the polynomial ring

$$
\mathbb{Z}\left[\sigma_{1}^{1}, \ldots, \sigma_{n_{1}}^{1}, \sigma_{1}^{2}, \ldots, \sigma_{n_{2}-n_{1}}^{2}, \ldots, \sigma_{1}^{k+1}, \ldots, \sigma_{n-n_{k}}^{k+1}\right]
$$

by $\mathbb{Z}[\sigma]$. With this notation, we can state another classical result, due to A. Borel 
ON QUANTUM COHOMOLOGY RINGS OF PARTIAL FLAG VARIETIES

Theorem 1.3. There is a canonical isomorphism $\mathbb{Z}[\sigma] / I \cong H^{*}(F)$, where $I$ is the ideal generated by $g_{1}^{k+1}, \ldots, g_{n}^{k+1}$.

\subsection{Classical Schubert Calculus for $F$.}

Let us recall the Schubert polynomials of Lascoux and Schützenberger ([LS1], [LS2]). Define operators $\partial_{i}, i=1, \ldots, n-1$ on $\mathbb{Z}\left[x_{1}, \ldots, x_{n}\right]$ by

$$
\partial_{i} P=\frac{P\left(x_{1}, \ldots, x_{n}\right)-P\left(x_{1}, \ldots, x_{i-1}, x_{i+1}, x_{i}, x_{i+2}, \ldots, x_{n}\right)}{x_{i}-x_{i+1}} .
$$

For any $w \in S_{n}$, write $w=w_{\circ} \cdot s_{i_{1}} \cdot \ldots \cdot s_{i_{k}}$, with $k=\frac{n(n-1)}{2}-\ell(w)$, where $s_{i}=(i, i+1)$ is the transposition interchanging $i$ and $i+1$, and $w_{\circ}$ is the permutation of longest length, given by $w_{\circ}(j)=n-j+1,1 \leq j \leq n$. The Schubert polynomial $\mathfrak{S}_{w}(x) \in \mathbb{Z}\left[x_{1}, \ldots, x_{n}\right]$ is defined by

$$
\mathfrak{S}_{w}(x)=\partial_{i_{k}} \circ \cdots \circ \partial_{i_{1}}\left(x_{1}^{n-1} x_{2}^{n-2} \ldots x_{n-1}\right) .
$$

It is shown in $[\mathrm{M}]$ that if $w \in S$, then the corresponding Schubert polynomial is symmetric in each group of variables

$$
\underbrace{x_{1} \ldots, x_{n_{1}}}, \underbrace{x_{n_{1}+1}, \ldots, x_{n_{2}}}, \ldots, \underbrace{x_{n_{k}+1}, \ldots, x_{n}}
$$

hence it can be written as a polynomial $P_{w}(\sigma)$, of weighted degree $\ell(w)$. We will call these $P_{w}(\sigma)$ Giambelli polynomials. The following theorem is due to BernsteinGelfand-Gelfand ([BGG]), and Demazure ([D]).

Theorem 1.4 (Giambelli-type formula). $\left[\Omega_{w}\right]=P_{w}(\sigma)$ in $H^{*}(F)$.

In particular, consider the cyclic permutations (of length $i$ ) $\alpha_{i, j}:=s_{n_{j}-i+1} \cdot \ldots$. $s_{n_{j}}$ and $\beta_{i, j}:=s_{n_{j}+i-1} \cdot \ldots \cdot s_{n_{j}}$. Note that these permutations are in $S$. Their Schubert polynomials are $\mathfrak{S}_{\alpha_{i, j}}=e_{i}^{n_{j}}$ and $\mathfrak{S}_{\beta_{i, j}}=h_{i}^{n_{j}}$, where $h_{i}^{n_{j}}$ is the $i^{\text {th }}$ complete symmetric polynomial in variables $x_{1}, \ldots, x_{n_{j}}$. Let $f_{i}^{j}(\sigma)$ be the polynomial in the $\sigma$-variables obtained from $h_{i}^{n_{j}}$. By Theorem 1.3,

$$
\left[\Omega_{\alpha_{i, j}}\right]=g_{i}^{j} \text { and }\left[\Omega_{\beta_{i, j}}\right]=f_{i}^{j} \text { in } H^{*}(F) \text {. }
$$

The following Pieri-type formula, due to A. Lascoux and M. Schützenberger [LS1], has been given recently a geometric proof by F. Sottile [S]:

Let $w, w^{\prime} \in S$. For $1 \leq a<b \leq n$ denote by $t_{a b}$ the transposition interchanging $a$ and $b$. Write $w \stackrel{\alpha_{i, j}}{\longrightarrow} w^{\prime}$ if there exist integers $a_{1}, b_{1}, \ldots, a_{i}, b_{i}$, satisfying

(1) $a_{m} \leq n_{j}<b_{m}$, for $1 \leq m \leq i$ and $w^{\prime}=w \cdot t_{a_{1} b_{1}} \cdot \ldots \cdot t_{a_{i} b_{i}}$;

(2) $\ell\left(w \cdot t_{a_{1} b_{1}} \cdot \ldots \cdot t_{a_{m} b_{m}}\right)=\ell(w)+m, 1 \leq m \leq i$;

$(3 \alpha)$ the integers $a_{1}, \ldots, a_{i}$ are distinct.

Similarly, $w \stackrel{\beta_{i, j}}{\longrightarrow} w^{\prime}$ if there exist $a_{1}, b_{1}, \ldots, a_{i}, b_{i}$ as above, satisfying $(1),(2)$ and 
Theorem 1.5 (Pieri-type formula). The following hold in $H^{*}(F)$ :

(i) $\left[\Omega_{\alpha_{i, j}}\right] \cdot\left[\Omega_{w}\right]=\sum_{w \stackrel{\alpha_{i, j}}{\longrightarrow} w^{\prime}}\left[\Omega_{w^{\prime}}\right]$.

(ii) $\left[\Omega_{\beta_{i, j}}\right] \cdot\left[\Omega_{w}\right]=\sum_{w \underset{\beta_{i, j}}{\longrightarrow} w^{\prime}}^{w}\left[\Omega_{w^{\prime}}\right]$.

Remark 1.6. From the exact sequence

$$
0 \longrightarrow L_{j} \longrightarrow Q_{j} \longrightarrow Q_{j-1} \longrightarrow 0
$$

we get

$$
c_{i}\left(Q_{j}\right)=\sum_{r=0}^{n_{j}-n_{j-1}} \sigma_{r}^{j} c_{i-r}\left(Q_{j-1}\right) .
$$

But one easily sees that $c_{i}\left(Q_{j}\right)=\left[\Omega_{\alpha_{i, j}}\right]$. Using (1.1), it follows that the polynomials $g_{i}^{j}$ satisfy the following recurssion (which in fact defines them uniquely):

$$
g_{i}^{j}=\sum_{r=0}^{n_{j}-n_{j-1}} \sigma_{r}^{j} g_{i-r}^{j-1}
$$

where, by convention, we set $g_{0}^{j-1}=1$ and $g_{m}^{j-1}=0$, if either $m<0$, or $m>n_{j-1}$.

Also, using the same exact sequence, the relations (1.1), (1.2), and the well known identity

$$
\left(\sum_{r=0}^{n_{j-1}} e_{r}^{n_{j-1}} t^{r}\right)^{-1}=\sum_{p \geq 0}(-1)^{p} h_{p}^{n_{j-1}} t^{p}
$$

we obtain that the following identity holds in $H^{*}(F)$ :

$$
\left[\Omega_{\alpha_{i, j}}\right]=\sum_{r=0}^{n_{j}-n_{j-1}}\left(\sum_{p=0}^{r}(-1)^{p}\left[\Omega_{\beta_{p, j-1}}\right] \cdot\left[\Omega_{\alpha_{r-p, j}}\right]\right) \cdot\left[\Omega_{\alpha_{i-r, j-1}}\right]
$$

where, by convention, $\left[\Omega_{\alpha_{0, m}}\right]=\left[\Omega_{\beta_{0, m}}\right]=1$ and $\left[\Omega_{\alpha_{<0, m}}\right]=0$, for all $m$.

\section{The Small Quantum Cohomology Ring of $F$}

We give below the precise definition of the small quantum cohomology ring only for the specific case of a partial flag manifold.

The 3-point, genus 0, Gromov-Witten $(G W)$ invariants of $F$, which we denote by $I_{3, \beta}\left(\gamma_{1} \gamma_{2} \gamma_{3}\right)$, are defined as intersection numbers on Kontsevich's moduli space of stable maps $\bar{M}_{0,3}(F, \beta)$ (see $[\mathrm{KM}]$, [Kon], [BM], [FP]). Here $\beta \in H_{2}(F)$ and $\gamma_{1}, \gamma_{2}, \gamma_{3} \in H^{*}(F)$. These numbers have the following enumerative significance $([\mathrm{FP}])$ :

Let $\Gamma_{1}, \Gamma_{2}, \Gamma_{3}$ be subvarieties of $F$, representing the cohomology classes $\gamma_{1}, \gamma_{2}, \gamma_{3}$ respectively. Let $g_{1}, g_{2}, g_{3} \in S L(n, \mathbb{C})$ be general elements, and denote by $g_{i} \Gamma_{i}$ the translate of $\Gamma_{i}$ by $g_{i}$. Then $I_{3, \beta}\left(\gamma_{1} \gamma_{2} \gamma_{3}\right)$ is the number of maps $\mu: \mathbb{P}^{1} \rightarrow F$ such that $\mu_{*}\left[\mathbb{P}^{1}\right]=\beta$ and $\mu\left(\mathbb{P}^{1}\right)$ meets $g_{1} \Gamma_{1}, g_{2} \Gamma_{2}$ and $g_{3} \Gamma_{3}$.

Since we will give a different construction of these invariants in Section 4, we will not say more about them here. The multiplication in the (small) quantum cohomology ring is defined using these $I_{3, \beta}$ as structure constants. More precisely, 
Introduce formal variables $q_{1}, \ldots, q_{k}$, corresponding respectively to the generators (cf. Theorem 1.1) $\left[\Omega_{\check{s}_{n_{1}}}\right], \ldots,\left[\Omega_{\breve{s}_{n_{k}}}\right]$ of $H_{2}(F)$. For a (holomorphic) map $\mu: \mathbb{P}^{1} \rightarrow F$ we can write $\beta=\mu_{*}\left[\mathbb{P}^{1}\right]=\sum_{j=1}^{k} d_{j}\left[\Omega_{\breve{s}_{n_{j}}}\right]$, with $d_{j}$ nonnegative integers. We will say that $\mu$ has multidegree $\bar{d}=\left(d_{1}, \ldots, d_{k}\right)$, and we'll replace $\beta$ by $\bar{d}$ in the notation for GW invariants.

Let $K:=\mathbb{Z}\left[q_{1} \ldots, q_{k}\right]$. On the $K$-module $H^{*}(F) \otimes_{\mathbb{Z}} K$, define the quantum multiplication $*$ by putting first

$$
\left[\Omega_{u}\right] *\left[\Omega_{v}\right]:=\sum_{\bar{d}} q_{1}^{d_{1}} \ldots q_{k}^{d_{k}} \sum_{w \in S} I_{3, \bar{d}}\left(\left[\Omega_{u}\right]\left[\Omega_{v}\right]\left[\Omega_{w}\right]\right)\left[\Omega_{\check{w}}\right]
$$

for all $u, v \in S$, and then extending linearly on $H^{*}(F)$ and trivially on $K$. The following theorem is a particular case of the general results on associativity of quantum cohomology ([B], [BM], [KM], [LT1], [LT2], [MS], [RT]).

Theorem 2.1. The operation $*$ defines an associative and commutative $K$-algebra structure on $H^{*}(F) \otimes_{\mathbb{Z}} K$.

$H^{*}(F) \otimes_{\mathbb{Z}} K$ together with this multiplication is called the small quantum cohomology ring of $F$, and denoted by $Q H^{*}(F)$. The goal of this paper is to give a description analogous to that in Section 1 for this new algebra.

\section{Quantum Schubert Calculus}

\subsection{The quantum version of the Pieri-type formula.}

We introduce first some notation. For integers $h, l$ satisfying $1 \leq h \leq l \leq k$, consider the cyclic permutations $\gamma_{h, l}:=s_{n_{h}} \cdot \ldots \cdot s_{n_{l+1}-1}$ and $\delta_{h, l}:=s_{n_{l}-1} \cdot \ldots$. $s_{n_{h-1}+1}$. Let now $1 \leq j \leq k$ and $1 \leq i \leq n_{j}$ be fixed, and let

$$
m \leq i, \quad 1 \leq h_{1}<\cdots<h_{m} \leq j \leq l_{m}<\cdots<l_{1} \leq k
$$

be integers. We denote by $\mathbf{h}$ and $\mathbf{l}$, respectively the collections $h_{1}, \ldots, h_{m}$ and $l_{1}, \ldots, l_{m}$. Let

$$
\begin{gathered}
\gamma_{\mathbf{h l}}:=\gamma_{h_{m}, l_{m}} \cdot \gamma_{h_{m-1}, l_{m-1}} \cdot \ldots \cdot \gamma_{h_{1}, l_{1}}, \\
\delta_{\mathbf{h l}}:=\delta_{h_{1}, l_{1}} \cdot \delta_{h_{2}, l_{2}} \cdot \ldots \cdot \delta_{h_{m}, l_{m}}
\end{gathered}
$$

Denote by $q_{\mathbf{h l}}$ the monomial

$$
q_{\mathbf{h l}}:=q_{h_{1}} \ldots q_{h_{2}-1} q_{h_{2}}^{2} \ldots q_{h_{3}-1}^{2} \ldots q_{h_{m}}^{m} \ldots q_{l_{m}}^{m} q_{l_{m}-1}^{m-1} \ldots q_{l_{m-1}}^{m-1} \ldots q_{l_{2}-1} \ldots q_{l_{1}} .
$$

Theorem 3.1 (Quantum Pieri formula). For every $1 \leq j \leq k, 1 \leq i \leq n_{j}$ and $w \in S$,

$$
\left[\Omega_{\alpha_{i, j}}\right] *\left[\Omega_{w}\right]=\sum_{\substack{\alpha_{i, j} \\ w \stackrel{w^{\prime}}{~}}}\left[\Omega_{w^{\prime}}\right]+\sum_{\mathbf{h}, \mathbf{l}} q_{\mathbf{h l}}\left(\sum_{w^{\prime \prime}}\left[\Omega_{w^{\prime \prime} \cdot \delta_{\mathbf{h l}}}\right]\right)
$$

where the second sum is over all collections $\mathbf{h}, \mathbf{l}$ such that

$$
\ell\left(w \cdot \gamma_{\mathbf{h l}}\right)=\ell(w)-\sum^{m}\left(n_{l_{c}+1}-n_{h_{c}}\right)
$$


while the last sum is over all permutations $w^{\prime \prime} \in S_{n}$ satisfying $w \cdot \gamma_{\mathbf{h l}} \stackrel{\tilde{\alpha}_{i, j}}{\longrightarrow} w^{\prime \prime}$, with $\tilde{\alpha}_{i, j}=\alpha_{i, j} \cdot s_{n_{j}} \cdot s_{n_{j}-1} \cdot \ldots \cdot s_{n_{j}-m+1}$, and

$$
\ell\left(w^{\prime \prime} \cdot \delta_{\mathbf{h l}}\right)=\ell\left(w^{\prime \prime}\right)-m-\sum_{c=1}^{m}\left(n_{l_{c}}-n_{h_{c}-1}\right) .
$$

Remark 3.2. (i) The first term in the right-hand side of the formula is the "classical" one, given by Theorem 1.5.

(ii) The condition

$$
\ell\left(w \cdot \gamma_{\mathbf{h l}}\right)=\ell(w)-\sum_{c=1}^{m}\left(n_{l_{c}+1}-n_{h_{c}}\right),
$$

can be rephrased equivalently as

$$
w\left(n_{h_{c}}\right)>\max \left\{w\left(n_{h_{c}}+1\right), \ldots, w\left(n_{l_{c}+1}\right)\right\}, \quad 1 \leq c \leq m .
$$

(iii) Note that if $m<i$, then $\tilde{\alpha}_{i, j}=s_{n_{j}-i+1} \cdot \ldots \cdot s_{n_{j}-m}$ gives the same kind of cyclic permutation as $\alpha_{i, j}$, but it determines a Schubert variety only on the flag varieties for which one of the quotients has rank $n_{j}-m$ !! In fact, as it will be seen in the proof of the Theorem, the last sum comes from applying Theorem 1.5 on a flag variety as above. It can be easily checked however, that the permutations $w^{\prime \prime} \cdot \delta_{\mathbf{h l}}$ are in fact in $S$, i.e. , they define Schubert varieties on our original $F\left(n_{1}, \ldots, n_{k}, V\right.$ ). (If $m=i$, then $\tilde{\alpha}_{i, j}$ is the identity permutation.) Also note that for the terms appearing in the last sum we have $\ell\left(w^{\prime \prime} \cdot \delta_{\mathbf{h l}}\right)=\ell(w)+i-\sum_{c=1}^{m}\left(n_{l_{c}}-n_{h_{c}-1}\right)-\sum_{c=1}^{m}\left(n_{l_{c}+1}-n_{h_{c}}\right)$.

(iv) In the case when $F$ is the complete flag variety, a Quantum Pieri formula is stated in the recent preprint [KiMa] of Kirillov and Maeno, and an algebraic proof is suggested. Their formulation is quite different, and we have not checked if it agrees with what Theorem 3.1 above says in that case.

We will prove the above Theorem in Section 6 . For the moment, let us see what it says in some special cases.

- Grassmannians: Let $k=1, n_{1}=m$, i.e., $F=G(m, n)$, the Grassmannian of $m$-dimensional quotients of $V$. Let $w$ be a Grassmannian permutation of descent $m$ and shape $\lambda=\left(\lambda_{1}, \ldots, \lambda_{m}\right)$, with $n-m \geq \lambda_{1} \geq \lambda_{2} \geq \cdots \geq \lambda_{m} \geq 0$. The partition $\lambda$ is defined by $\lambda_{m-j+1}=w(j)-j$. Denote $\Omega_{\lambda}:=\Omega_{w}$. In particular, the subvariety $\Omega_{\alpha_{i, 1}}$ is $\Omega_{\left(1^{i}, 0^{m-i}\right)}$ with the new notation. The following result is due to A. Bertram $([\mathrm{Be}])$

Corollary 3.3 (Quantum Pieri for Grassmannians). The following holds in $Q H^{*}(G(m, n))$ :

$$
\left[\Omega_{\left(1^{i}, 0^{m-i}\right)}\right] *\left[\Omega_{\lambda}\right]=(\text { classical term })+q\left(\sum_{\mu}\left[\Omega_{\mu}\right]\right),
$$

where $\mu$ ranges over partitions with at most $m$ parts, satisfying $|\mu|=|\lambda|+i-n$, and $\lambda_{1}-1 \geq \mu_{1} \geq \lambda_{2}-1 \geq \cdots \geq \lambda_{m}-1 \geq \mu_{m} \geq 0$.

- Complete flag varieties: Let $k=n-1$, hence $n_{j}=j$ for all $j$, i.e., $F=$ $F(1,2, \ldots, n-1, V)$. In the case $i=1$, we have $\alpha_{1, j}=s_{j}$ and (3.1) specializes 
Corollary 3.4 (Quantum Monk formula). One has in $Q H^{*}(F)$

$$
\left[\Omega_{s_{j}}\right] *\left[\Omega_{w}\right]=(\text { classical term })+\sum_{t_{h l}} q_{h} \ldots q_{l-1}\left[\Omega_{w \cdot t_{h l}}\right]
$$

where the sum is over all transposition of integers $h, l$, with $1 \leq h \leq j<l \leq n$, such that $\ell\left(w \cdot t_{h l}\right)=\ell(w)-2(l-h)+1$.

Note, however, that even for the case of complete flags, Theorem 3.1 says much more than Corollary 3.4 !

Finally, we look now closer to a special case, which will be needed later. Recall the identity (1.3), which holds in the classical cohomology ring of our partial flag variety:

$$
\left[\Omega_{\alpha_{i, j}}\right]=\sum_{r=0}^{n_{j}-n_{j-1}}\left(\sum_{p=0}^{r}(-1)^{p}\left[\Omega_{\beta_{p, j-1}}\right] \cdot\left[\Omega_{\alpha_{r-p, j}}\right]\right) \cdot\left[\Omega_{\alpha_{i-r, j-1}}\right] .
$$

We want to compute the right-hand side when the classical product is replaced by the quantum product. Of course, the answer is obtained by applying Theorem 3.1 twice, but this would seem to give, besides the classical term $\left[\Omega_{\alpha_{i, j}}\right]$, lots of "quantum correction" terms. In fact, a more careful analysis will show that there is either no correction term, or only one such term which we identify explicitely. It is better to break the computation into two pieces.

Lemma 3.5. (i) In the classical cohomology ring $H^{*}(F)$, we have for $0 \leq p \leq r \leq$ $n_{j}-n_{j-1}$

$$
\left[\Omega_{\beta_{p, j-1}}\right] \cdot\left[\Omega_{\alpha_{r-p, j}}\right]=\left[\Omega_{\beta_{p, j-1} \cdot \alpha_{r-p, j}}\right] .
$$

(ii) In the quantum cohomology ring $Q H^{*}(F)$ we have

$$
\left[\Omega_{\beta_{p, j-1}}\right] *\left[\Omega_{\alpha_{r-p, j}}\right]=\left[\Omega_{\beta_{p, j-1} \cdot \alpha_{r-p, j}}\right]
$$

as well, i.e., there are no quantum correction terms.

Proof. $(i)$ is a straightforward computation, e.g., using Sottile's Theorem 1.5.

(ii) Pick $1 \leq h_{1}<\cdots<h_{m} \leq j \leq l_{m}<\cdots<l_{1} \leq k$. Since $n_{j} \geq r+n_{j-1} \geq p+$ $n_{j-1}$, we have also $n_{l_{1}+1} \geq n_{j+1} \geq n_{j}+1 \geq n_{j-1}+p+1$. Therefore $\beta_{p, j-1}\left(n_{l_{1}+1}\right)>$ $\beta_{p, j-1}(m)$ for all $m<n_{l_{1}+1}$, by the definition of $\beta_{p, j-1}$. In particular

$$
\beta_{p, j-1}\left(n_{l_{1}+1}\right)>\beta_{p, j-1}\left(n_{h_{1}}\right) .
$$

To get a quantum contribution for the chosen $h_{i}$ and $l_{i}$, we should have necessarily, by Remark $3.2(i i)$,

$$
\beta_{p, j-1}\left(n_{h_{1}}\right)>\max \left\{\beta_{p, j-1}\left(n_{h_{1}}+1\right), \ldots, \beta_{p, j-1}\left(n_{l_{1}+1}\right)\right\}
$$

This contradicts (3.2). 
Lemma 3.6. The product $\left[\Omega_{\beta_{p, j-1} \cdot \alpha_{r-p, j}}\right] *\left[\Omega_{\alpha_{i-r, j-1}}\right]$ has no quantum correction terms, unless $r=p=n_{j}-n_{j-1}$ and $i \geq n_{j}-n_{j-2}$, in which case there is exactly one such term, namely $q_{j-1}\left[\Omega_{\alpha_{i-\left(n_{j}-n_{j-2}\right), j-2}}\right]$.

Proof. This time we need to pick $1 \leq h_{1}<\cdots<h_{m} \leq j-1 \leq l_{m}<\cdots<l_{1} \leq k$. If any of the $h_{i}$, or $l_{i}$ are different from $j-1$, the condition

$$
w\left(n_{h_{i}}\right)>\max \left\{w\left(n_{h_{i}}+1\right), \ldots, w\left(n_{l_{i}+1}\right)\right\}
$$

of Remark $3.2(i i)$ is easily seen to be contradicted for $w:=\beta_{p, j-1} \cdot \alpha_{r-p, j}$. Hence $m=1, h_{1}=l_{1}=j-1$, and we need

$$
w\left(n_{j-1}\right)>\max \left\{w\left(n_{j-1}+1\right), \ldots, w\left(n_{j}\right)\right\} .
$$

This happens iff $r=p=n_{j}-n_{j-1}$, i.e., the only case that may give quantum contributions is the product

$$
\left[\Omega_{\beta_{n_{j}-n_{j-1}, j-1}}\right] *\left[\Omega_{\alpha_{i-\left(n_{j}-n_{j-1}\right), j-1}}\right],
$$

for $h=l=j-1$. In this case $\beta_{n_{j}-n_{j-1}, j-1} \cdot \gamma_{h, l}=i d$ (the identity permutation), and the Quantum Pieri formula (3.1) specializes to give the Lemma.

From the identity (1.3) and the two previous Lemmas we get immediately

Corollary 3.7. The following identity holds in $Q H^{*}(F)$ :

$$
\begin{aligned}
& \sum_{r=0}^{n_{j}-n_{j-1}}\left(\sum_{p=0}^{r}(-1)^{p}\left[\Omega_{\beta_{p, j-1}}\right] *\left[\Omega_{\alpha_{r-p, j}}\right]\right) *\left[\Omega_{\alpha_{i-r, j-1}}\right]= \\
& {\left[\Omega_{\alpha_{i, j}}\right]+(-1)^{n_{j}-n_{j-1}} q_{j-1}\left[\Omega_{\alpha_{i-\left(n_{j}-n_{j-2}\right), j-2}}\right] .}
\end{aligned}
$$

\subsection{The Quantum Giambelli formula.}

Definition 3.8. For $0 \leq j \leq k+1,1 \leq i \leq n_{j}$, let $G_{i}^{j} \in \mathbb{Z}[\sigma, q]$ be the polynomials defined in one of the following equivalent ways.

(3.8.1) Set $G_{0}^{j}:=1, G_{1}^{j}:=g_{1}^{j}$, for all $j$. Then $G_{i}^{j}$ for $i \geq 2$, and all $j$ is defined recursively by

$$
G_{i}^{j}:=(-1)^{n_{j}-n_{j-1}+1} q_{j-1} G_{i-\left(n_{j}-n_{j-2}\right)}^{j-2}+\sum_{r=0}^{n_{j}-n_{j-1}} \sigma_{r}^{j} G_{i-r}^{j-1} .
$$

(3.8.2) For each $1 \leq j \leq k+1$, construct a graph as follows:

- choose $j$ vertices and label them $\mathbf{v}_{1}, \ldots, \mathbf{v}_{j}$;

- for every $1 \leq l \leq j-1$, join the vertices $\mathbf{v}_{l}$ and $\mathbf{v}_{l+1}$ by an edge and give it the label $(-1)^{n_{l+1}-n_{l}+1} q_{l}$;

- for every $1 \leq l \leq j$, attach $n_{l}-n_{l-1}$ tails to the vertex $\mathbf{v}_{l}$, with labels 
Now define $G_{i}^{j}$ to be the sum of all monomials obtained by choosing edges in this graph and forming the product of their labels, such that the total degree of the monomial is $i$, where $\operatorname{deg}\left(q_{l}\right)=n_{l+1}-n_{l-1}$ and $\operatorname{deg}\left(\sigma_{m}^{l}\right)=m$, for every $l, m$, and no two of the chosen edges share a common vertex. This description has been shown to us by W. Fulton.

(3.8.3) For each $j$, the polynomials $G_{i}^{j}, 1 \leq i \leq n_{j}$ are the coefficients of the characteristic polynomial $\operatorname{det}\left(A_{n_{j}}^{q}+\lambda I\right)$, where $A_{n_{j}}^{q}$ is the upper left $n_{j} \times n_{j}$ submatrix of the Astashkevich-Sadov-Kim matrix $A_{n}^{q}$ ([AS], [Kim1], [Kim 2]).

It is immediate from any of these descriptions that $G_{i}^{j}(\sigma, 0)=g_{i}^{j}(\sigma)$. We are now ready to formulate a special case of the Quantum Giambelli formula.

Theorem 3.9. $(i)\left[\Omega_{\alpha_{i, j}}\right]=G_{i}^{j}(\sigma, q)$ in $Q H^{*}(F)$, for all $1 \leq i \leq n_{j}, 0 \leq j \leq k$.

(ii) $G_{i}^{k+1}(\sigma, q)=0$ in $Q H^{*}(F)$, for all $1 \leq i \leq n$.

Proof. Induction on $j$, using the classical Giambelli formula, the recursion (1.2) satisfied by the $g_{i}^{j}$ 's, the identity (1.3), Corollary 3.7, and the recursion (3.8.1) satisfied by the $G_{i}^{j}$ 's (cf. the proof of Theorem $5.6(i)$ in [C-F2] for the case of complete flags).

Corollary 3.10 ([AS], [Kim1], [Kim2]). There is a canonical isomorphism

$$
Q H^{*}(F) \cong \mathbb{Z}[\sigma, q] / I_{q},
$$

where $I_{q}$ is the ideal $\left(G_{1}^{k+1}, \ldots, G_{n}^{k+1}\right)$.

Proof. Follows from Theorem 3.9 (ii) and [ST, Theorem 2.2].

Remark 3.11. Theorem $3.9(i i)$ and Corollary 3.10 were formulated independently by A. Astashkevich and V. Sadov $([\mathrm{AS}])$, and B. Kim $([\operatorname{Kim} 1])$, with the proof completed in [Kim2]. As far as I know, Theorem $3.9(i)$ is new here. For the case of complete flags, Theorem 3.9 and Corollary 3.10 were proved first in [C-F1].

Following [FGP], Theorem 3.9, together with a quantum analogue of Theorem 1.2 are sufficient to obtain the general Quantum Giambelli formula. More precisely, this goes as follows:

For a partition $\Lambda_{j}:=\left(\lambda_{j, 1}, \ldots, \lambda_{j, n_{j+1}-n_{j}}\right)$ with (at most) $n_{j+1}-n_{j}$ parts and such that each part $\lambda_{j, m}$ is at most $n_{j}$, set

$$
g_{\Lambda_{j}}^{(j)}:=g_{\lambda_{j, 1}}^{j} g_{\lambda_{j, 2}}^{j} \ldots g_{\lambda_{j, n_{j+1}-n_{j}}^{j}}^{j} .
$$

Define "standard elementary monomials" $g_{\Lambda}:=g_{\Lambda_{1} \Lambda_{2} \ldots \Lambda_{k}} \in \mathbb{Z}[\sigma]$ by

$$
g_{\Lambda}:=g_{\Lambda_{1}}^{(1)} g_{\Lambda_{2}}^{(2)} \ldots g_{\Lambda_{k}}^{(k)} .
$$

The number of such monomials is

$$
\sharp\left\{g_{\Lambda}\right\}=\prod_{j=0}^{k}\left(\begin{array}{c}
n_{j+1} \\
n_{j}
\end{array}\right),
$$

which coincides with the rank of $H^{*}(F)$. It follows by realizing $F$ as a succesion of Grassmann bundles that the monomials $\left\{g_{\Lambda}\right\}$ generate $H^{*}(F)$ over $\mathbb{Z}$. Summarizing, 
Proposition 3.12. The standard elementary monomials $\left\{g_{\Lambda}\right\}$ form a linear basis in $H^{*}(F)$.

Since the Giambelli polynomials $\left\{P_{w}(\sigma)\right\}_{w \in S}$ also form a basis in $H^{*}(F)$, we can write uniquely

$$
P_{w}=\sum_{\Lambda} a_{\Lambda} g_{\Lambda}
$$

with $a_{\Lambda}$ integers (depending, of course, on $w$ ). The following definition was given in the case of complete flags in [FGP].

Definition 3.13. The standard quantum elementary monomial $G_{\Lambda}$ is the polynomial in $\mathbb{Z}[\sigma, q]$ obtained by replacing in $g_{\Lambda}$ each factor $g_{\lambda}^{j}$ by the corresponding $G_{\lambda}^{j}$, defined in 3.8 .

The quantum Giambelli polynomial $P_{w}^{q} \in \mathbb{Z}[\sigma, q]$ is defined by

$$
P_{w}^{q}:=\sum_{\Lambda} a_{\Lambda} G_{\Lambda}
$$

with $a_{\Lambda}$ the integers from (3.4).

The following is immediate from the definitions 3.8 and 3.13.

Proposition 3.14. (i) $P_{w}^{q}(\sigma, q)$ is a weighted homogeneous polynomial of weighted degree $\ell(w)$, where $\sigma_{i}^{j}$ has degree $i$ and $q_{j}$ has degree $n_{j+1}-n_{j-1}$, for all $1 \leq j \leq k$.

(ii) $P_{w}^{q}(\sigma, 0)=P_{w}(\sigma)$

(iii) (cf. [FGP, 3.6-3.7]) $\left\{G_{\Lambda}\right\}$ and $\left\{P_{w}^{q}\right\}$ are linear bases for $Q H^{*}(F)$.

Let $w^{\circ} \in S$ be the longest element, given by $w(i)=n-n_{j}+i-n_{j-1}$, for all $n_{j-1}+1 \leq i \leq n_{j}, 1 \leq j \leq k+1$. Its length is $\ell\left(w^{\circ}\right)=\sum_{j=1}^{k}\left(n-n_{j}\right)\left(n_{j}-n_{j-1}\right)=$ $\operatorname{dim} F$, and $\left[\Omega_{w^{\circ}}\right]$ is the class of a point in $H_{0}(F)$. By the classical Giambelli formula,

$$
\left[\Omega_{w^{\circ}}\right]=P_{w^{\circ}}(\sigma)=\left(\sigma_{n_{1}}^{1}\right)^{n-n_{1}}\left(\sigma_{n_{2}-n_{1}}^{2}\right)^{n-n_{2}} \ldots\left(\sigma_{n_{k}-n_{k-1}}\right)^{n-n_{k}},
$$

while expressing $\left[\Omega_{w^{\circ}}\right]$ in the basis $\left\{g_{\Lambda}\right\}$ yields

$$
\left[\Omega_{w^{\circ}}\right]=\underbrace{g_{n_{1}}^{1} g_{n_{1}}^{1} \ldots g_{n_{1}}^{1} g_{n_{3}-n_{2}}^{2} g_{n_{2}}^{2} g_{n_{2}}^{2} \ldots g_{n_{2}}^{2}}_{n_{2}-n_{1} \text { factors }} \cdots \underbrace{g_{n_{k}}^{k} g_{n_{k}}^{k} \ldots g_{n_{k}}^{k}}_{n_{k+1}-n_{k} \text { factors }}=g_{\Lambda^{\circ}},
$$

where

$$
\Lambda^{\circ}=\left(\Lambda_{1}, \ldots, \Lambda_{k}\right), \Lambda_{j}=(\underbrace{n_{j}, \ldots, n_{j}}_{n_{j+1}-n_{j} \text { terms }}) .
$$

For a polynomial $R \in \mathbb{Z}[\sigma]$ consider the expansion of its coset $R(\bmod I) \in \mathbb{Z}[\sigma] / I$ in the basis $\left\{P_{w}\right\}$, and define

$$
\langle R\rangle:=\text { coefficient of } P_{w^{\circ}}
$$

Alternately, we can expand $R(\bmod I)$ in the basis $\left\{g_{\Lambda}\right\}$ and take the coefficient of $g_{\Lambda^{\circ}}$. By the classical Giambelli formula (Theorem 1.3), we can reformulate Theorem 
Proposition 3.15. The polynomials $\left\{P_{w}\right\}$ satisfy the following orthogonality property:

$$
\left\langle P_{w} P_{v}\right\rangle=\left\{\begin{array}{ll}
1, & \text { if } v=\check{w} \\
0, & \text { otherwise }
\end{array},\right.
$$

where $\check{w}$ is the permutation giving the Schubert variety dual to $\left[\Omega_{w}\right]$.

Similarly, for $R(\sigma, q) \in \mathbb{Z}[\sigma, q]$ consider the expansion of $R\left(\bmod I_{q}\right)$ in the basis $\left\{P_{w}^{q}\right\}$ (or $\left\{G_{\Lambda}\right\}$ respectively), and define

$$
\langle\langle R\rangle\rangle:=\text { coefficient of } P_{w^{\circ}}^{q}\left(\text { or } G_{\Lambda^{\circ}}\right. \text { respectively). }
$$

Theorem 3.16 (Orthogonality of the quantum Giambelli polynomials).

$$
\left\langle\left\langle P_{w}^{q} P_{v}^{q}\right\rangle\right\rangle=\left\{\begin{array}{ll}
1, & \text { if } v=\check{w} \\
0, & \text { otherwise }
\end{array} .\right.
$$

Proof. The proof will be given in Section 6 .

Remark 3.17. For the special case of complete flags, the above Theorem is due to $[\mathrm{FGP}]$, and was proved using combinatorial techniques. The proof we will give in this paper is geometric.

Theorem 3.18 (Quantum Giambelli formula). $\left[\Omega_{w}\right]=P_{w}^{q}(\sigma, q)$ in $Q H^{*}(F)$, for all $w \in S$.

Proof. Having established the special case of Quantum Giambelli (Theorem $3.9(i)$ ), and the orthogonality of the $P_{w}^{q}$ 's (Theorem 3.16), the proof of the Main Theorem in [FGP, Section 4] applies without changes in our more general case.

Corollary 3.19 (A. Bertram, [Be]). For the Grassmannian $G(m, n)$, the classical and quantum Giambelli formulae are the same.

Proof. By the definition 3.8, we have $G_{i}^{1}=g_{i}^{1}$, for all $1 \leq i \leq m$, hence the quantum Giambelli polynomials coincide with the classical ones.

We have completed the description of $Q H^{*}(F)$, modulo the proofs of Theorems 3.1 and 3.16. The last three sections of the paper are devoted to these proofs, which are based on the geometry of compactifications of spaces of maps $\mathbb{P}^{1} \rightarrow F$ given by hyperquot schemes. Most of the arguments in [C-F2], where the case of complete flags is treated, require little or no changes, therefore we will refer to the corresponding results in [C-F2] when appropriate, and give details only as needed.

\section{GW-INVARIANTS VIA HYPERQUOT SCHEMES}

We recall in this section the construction of 3-point, genus $0 \mathrm{GW}$-invariants by means of hyperquot schemes. Details can be found in [Be], [C-F2].

\subsection{Hom and hyperquot schemes.}

For fixed $\bar{d}=\left(d_{1}, d_{2}, \ldots, d_{k}\right)$, let 
be the moduli space of holomorphic maps $\mu: \mathbb{P}^{1} \rightarrow F$ of multidegree $\bar{d}$, i.e., such that $\mu_{*}\left[\mathbb{P}^{1}\right]=\sum_{j=1}^{k} d_{j}\left[\Omega_{\check{s}_{n_{j}}}\right]$.

Since $F$ is a homogeneous space, standard deformation theory shows that $H_{\bar{d}}$ is a smooth quasiprojective variety of dimension

$h^{0}\left(\mathbb{P}^{1}, \mu^{*} T_{F}\right)=\operatorname{dim} F-\mu_{*}\left[\mathbb{P}^{1}\right] \cdot\left(K_{F}\right)=\sum_{j=1}^{k}\left(n-n_{j}\right)\left(n_{j}-n_{j-1}\right)+\sum_{j=1}^{k} d_{j}\left(n_{j+1}-n_{j-1}\right)$.

To give a map of multidegree $\bar{d}$ is equivalent to specifying a sequence of quotient bundles

$$
V_{\mathbb{P}^{1}} \rightarrow M_{k} \rightarrow \cdots \rightarrow M_{1},
$$

with $\operatorname{rank} M_{j}=n_{j}, \operatorname{deg}\left(M_{j}\right)=d_{j}$, or, by dualizing, a sequence of subbundles

$$
S_{1} \subset \cdots \subset S_{k} \subset V_{\mathbb{P}^{1}}^{*}
$$

with $\operatorname{rank} S_{j}=n_{j}, \operatorname{deg}\left(S_{j}\right)=-d_{j}$. Let $T_{j}:=V_{\mathbb{P}^{1}}^{*} / S_{k-j+1}$. The Hilbert polynomial of $T_{j}$ is $P_{j}(m)=(m+1)\left(n-n_{k-j+1}\right)+d_{k-j+1}$.

Let $\mathcal{H} \mathcal{Q}_{\bar{d}}:=\mathcal{H} \mathcal{Q}_{P_{1}, \ldots, P_{k}}\left(\mathbb{P}^{1}, V_{\mathbb{P}^{1}}^{*}\right)$ be the hyperquot scheme parametrizing flagged sequences of quotient sheaves of $V_{\mathbb{P}^{1}}^{*}$, with Hilbert polynomials given by $P_{1}, \ldots, P_{k}$.

Theorem 4.1 ([Lau], [C-F1, C-F2], [Kim3]). (i) $\mathcal{H}_{\bar{d}}$ is a smooth, irreducible, projective variety, of dimension

$$
\sum_{j=1}^{k}\left(n-n_{j}\right)\left(n_{j}-n_{j-1}\right)+\sum_{j=1}^{k} d_{j}\left(n_{j+1}-n_{j-1}\right),
$$

containing $H_{\bar{d}}$ as an open dense subscheme.

(ii) $\mathcal{H Q}_{\bar{d}}$ is a fine moduli space, i.e., there exists an universal sequence

$$
V_{\mathbb{P}^{1} \times \mathcal{H Q}_{\bar{d}}}^{*} \rightarrow \mathcal{T}_{k}^{\bar{d}} \rightarrow \cdots \rightarrow \mathcal{T}_{2}^{\bar{d}} \rightarrow \mathcal{T}_{1}^{\bar{d}} \rightarrow 0
$$

on $\mathbb{P}^{1} \times \mathcal{H} \mathcal{Q}_{\bar{d}}$, such that each $\mathcal{T}_{j}^{\bar{d}}$ is flat over $\mathcal{H} \mathcal{Q}_{\bar{d}}$, with relative Hilbert polynomial $P_{j}(m)$, and having the following property:

For every scheme $X$ over $\mathbb{C}$, together with a sequence of quotients

$$
V_{\mathbb{P}^{1} \times X}^{*} \rightarrow Q_{k} \rightarrow \cdots \rightarrow Q_{1}
$$

such that each $Q_{j}$ is flat over $X$, with relative Hilbert polynomial $P_{j}$, there exists an unique morphism $\Phi_{X}: X \rightarrow \mathcal{H} \mathcal{Q}_{\bar{d}}$ such that the sequence (†) is the pull-back of $(\dagger)$ via $\left(i d, \Phi_{X}\right)$.

(iii) Let $\mathcal{S}_{j}^{\bar{d}}:=\operatorname{ker}\left(V_{\mathbb{P}^{1} \times \mathcal{H Q}_{\bar{d}}}^{*} \rightarrow \mathcal{T}_{k-j+1}^{\bar{d}}\right)$. Then $\mathcal{S}_{j}^{\bar{d}}$ is a vector bundle of rank $n_{j}$ and relative degree $-d_{j}$ on $\mathbb{P}^{1} \times \mathcal{H} \mathcal{Q}_{\bar{d}}$. 


\subsection{Generalized Schubert varieties on $H_{\bar{d}}$ and $\mathcal{H} \mathcal{Q}_{\bar{d}}$.}

The moduli space of maps comes with an universal "evaluation" morphism

$$
e v: \mathbb{P}^{1} \times H_{\bar{d}} \rightarrow F,
$$

given by $e v(t,[\mu])=\mu(t)$, which can be used to pull-back Schubert varieties to $H_{\bar{d}}$. More precisely, for $t \in \mathbb{P}^{1}, w \in S$, define a subscheme of $H_{\bar{d}}$ by

$$
\Omega_{w}(t)=e v^{-1}\left(\Omega_{w}\right) \bigcap\left(\{t\} \times H_{\bar{d}}\right) .
$$

(Set-theoretically, $\Omega_{w}(t)=\left\{[\mu] \in H_{\bar{d}} \mid \mu(t) \in \Omega_{w}\right\}$.)

Alternately, the pull-back $\Omega_{w}(t)$ of a Schubert variety can be described as the degeneracy locus

$$
\left\{\operatorname{rank}\left(V_{p} \otimes \mathcal{O} \rightarrow e v^{*} Q_{q}\right) \leq r_{w}(q, p), 1 \leq p \leq n, q \in\left\{n_{1}, \ldots, n_{k}\right\}\right\} \bigcap\left(\{t\} \times H_{\bar{d}}\right)
$$

where the $Q_{j}$ 's are the tautological quotient bundles on $F$, and $V_{1} \subset \cdots \subset V_{n-1} \subset$ $V_{n}=V$ is our fixed reference flag. This last description may be used to extend $\Omega_{w}(t)$ to $\mathcal{H} \mathcal{Q}_{\bar{d}}$.

Definition 4.3. $\bar{\Omega}_{w}(t)$ is the subscheme of $\mathcal{H} \mathcal{Q}_{\bar{d}}$ defined as the degeneracy locus $\left\{\operatorname{rank}\left(V_{p} \otimes \mathcal{O} \rightarrow\left(\mathcal{S}_{q}^{\bar{d}}\right)^{*} \leq r_{w}(q, p), 1 \leq p \leq n, q \in\left\{n_{1}, \ldots, n_{k}\right\}\right\} \bigcap\left(\{t\} \times \mathcal{H} \mathcal{Q}_{\bar{d}}\right)\right.$

\subsection{GW-invariants.}

To define the GW-invariants, we need the following "moving lemma"-type result

Theorem 4.4 (Moving Lemma). (i) Let $Y$ be a fixed subvariety of $H_{\bar{d}}$. For any $w \in S$, a corresponding general translate of $\Omega_{w} \subset F$, and $t \in \mathbb{P}^{1}$, the intersection $Y \cap \Omega_{w}(t)$ is either empty, or has pure codimension $\ell(w)$ in $Y$. In particular, for any $w_{1}, \ldots, w_{N} \in S ; t_{1}, \ldots, t_{N} \in \mathbb{P}^{1}$, and general translates of $\Omega_{w_{i}} \subset F$, the intersection $\bigcap_{i=1}^{N} \Omega_{w_{i}}\left(t_{i}\right)$ is either empty, or has pure codimension $\sum_{i=1}^{N} \ell\left(w_{i}\right)$ in $H_{\bar{d}}$.

(ii) Moreover, if $t_{1}, \ldots, t_{N}$ are distinct, then for general translates of the $\Omega_{w_{i}}$ the intersection $\bigcap_{i=1}^{N} \bar{\Omega}_{w_{i}}\left(t_{i}\right)$ is either empty, or has pure codimension $\sum_{i=1}^{N} \ell\left(w_{i}\right)$ in $\mathcal{H}_{\mathcal{Q}_{\bar{d}}}$ and is the Zariski closure of $\bigcap_{i=1}^{N} \Omega_{w_{i}}\left(t_{i}\right)$.

Proof. (i) This follows from a Theorem of Kleiman ([Kl]), since $F$ is a homogeneous space.

(ii) The proof will be given in Section 6 .

In particular, $\bar{\Omega}_{w}(t)$ is the closure of $\Omega_{w}(t)$ in $\{t\} \times \mathcal{H} \mathcal{Q}_{\bar{d}}$, and has pure codimension $\ell(w)$, hence, via the identification $\{t\} \times \mathcal{H} \mathcal{Q}_{\bar{d}} \cong \mathcal{H} \mathcal{Q}_{\bar{d}}$, it determines a class $\left[\bar{\Omega}_{w}(t)\right] \in H^{2 \ell(w)}\left(\mathcal{H} \mathcal{Q}_{\bar{d}}\right)$. From the above Theorem we get immediately (see e.g. 
Corollary 4.5. The class $\left[\bar{\Omega}_{w}(t)\right]$ in the cohomology (or Chow) ring of $\mathcal{H} \mathcal{Q}_{\bar{d}}$ is independent of $t \in \mathbb{P}^{1}$ and the flag $V_{\bullet} \subset V$.

Corollary 4.6. Assume that $\sum_{i=1}^{N} \ell\left(w_{i}\right)=\operatorname{dim}\left(H_{\bar{d}}\right)$. Then, as long as $t_{1}, \ldots, t_{N}$ are distinct, and we pick general translates of the subvarieties $\Omega_{w_{i}} \subset F$, the number of points in $\bigcap_{i=1}^{N} \Omega_{w_{i}}\left(t_{i}\right)=\bigcap_{i=1}^{N} \bar{\Omega}_{w_{i}}\left(t_{i}\right)$ can be computed as the intersection number

$$
\int_{\mathcal{H} \mathcal{Q}_{\bar{d}}}\left[\bar{\Omega}_{w_{1}}\left(t_{1}\right)\right] \cup \cdots \cup\left[\bar{\Omega}_{w_{N}}\left(t_{N}\right)\right]
$$

(hence it is independent of $t_{i}$ and the general translates of $\Omega_{w_{i}}$ ).

The corollaries imply that, for general translates of $\Omega_{w_{1}}, \ldots, \Omega_{w_{N}}$ and distinct $t_{1}, \ldots, t_{N}$, we have a well defined intersection number

$$
\left\langle\Omega_{w_{1}}, \ldots, \Omega_{w_{N}}\right\rangle_{\bar{d}}:= \begin{cases}\operatorname{card}\left(\bigcap_{i=1}^{N} \Omega_{w_{i}}\left(t_{i}\right)\right), & \text { if } \sum_{i=1}^{N} \ell\left(w_{i}\right)=\operatorname{dim}\left(H_{\bar{d}}\right) \\ 0, & \text { otherwise. }\end{cases}
$$

We will call this the Gromov-Witten invariant associated to the Schubert classes $\left[\Omega_{w_{1}}\right], \ldots,\left[\Omega_{w_{N}}\right]$.

Corollary 4.7. The invariant $I_{3, \bar{d}}\left(\left[\Omega_{w_{1}}\right]\left[\Omega_{w_{2}}\right]\left[\Omega_{w_{3}}\right]\right)$ defined using Kontsevich's space of stable maps $\bar{M}_{0,3}(F, \bar{d})$ coincides with $\left\langle\Omega_{w_{1}}, \Omega_{w_{2}}, \Omega_{w_{3}}\right\rangle_{\bar{d}}$.

Remark 4.8. By the preceding Corollary we have

$$
\left[\Omega_{u}\right] *\left[\Omega_{v}\right]=\sum_{\bar{d}} q_{1}^{d_{1}} \ldots q_{k}^{d_{k}} \sum_{w \in S}\left\langle\Omega_{u}, \Omega_{v}, \Omega_{w}\right\rangle_{\bar{d}}\left[\Omega_{\check{w}}\right]
$$

Note that we have also

$$
\left[\Omega_{w_{1}}\right] *\left[\Omega_{w_{2}}\right] * \cdots *\left[\Omega_{w_{N}}\right]=\sum_{\bar{d}} q_{1}^{d_{1}} \ldots q_{k}^{d_{k}} \sum_{w \in S}\left\langle\Omega_{w_{1}}, \ldots, \Omega_{w_{N}}, \Omega_{w}\right\rangle_{\bar{d}}\left[\Omega_{\check{w}}\right] .
$$

We will use (4.1) in Section 6, for the proof of Theorem 3.16.

Finally, we record for later use a generalization of (part of) Theorem 4.4, due to B. Kim ([Kim3, Corollary 3.2]).

For every irreducible closed subvariety $Y \subset F$ and every $t \in \mathbb{P}^{1}$, we denote by $Y(t)$ the preimage $e v^{-1}(Y) \bigcap\{y\} \times H_{\bar{d}}$ and by $\overline{Y(t)}$ the closure of $Y(t)$ in $\{y\} \times \mathcal{H} \mathcal{Q}_{\bar{d}}$.

Proposition 4.9. Let $Y_{1}, \ldots, Y_{N}$ be closed, irreducible subvarieties in $F$, and let $t_{1}, \ldots, t_{N}$ be distinct points in $\mathbb{P}^{1}$. Assume that $\sum_{i=1}^{N} \operatorname{codim} Y_{i}=\operatorname{dim} H_{\bar{d}}$. Then for general translates of $Y_{i}$, the intersection scheme $\bigcap_{i=1}^{N} Y_{i}\left(t_{i}\right)$ is either empty, or it consists of finitely many reduced points. Moreover,

$$
\bigcap_{i=1}^{N} Y_{i}\left(t_{i}\right)=\bigcap_{i=1}^{N} \overline{Y_{i}\left(t_{i}\right)}
$$

and the cardinality of this set is equal to the intersection number

$$
\int_{\mathcal{H} \mathcal{Q}_{\bar{d}}}\left[\overline{Y_{1}\left(t_{1}\right)}\right] \cup \cdots \cup\left[\overline{Y_{N}\left(t_{N}\right)}\right]
$$




\section{The Boundary of $\mathcal{H} \mathcal{Q}_{\bar{d}}$}

The space $H_{\bar{d}}$ is the largest subscheme of $\mathcal{H} \mathcal{Q}_{\bar{d}}$ with the property that on $\mathbb{P}^{1} \times H_{\bar{d}}$ the sheaf injections in the universal sequence

$$
0 \hookrightarrow \mathcal{S}_{1}^{\bar{d}} \hookrightarrow \mathcal{S}_{2}^{\bar{d}} \hookrightarrow \cdots \hookrightarrow \mathcal{S}_{k}^{\bar{d}} \hookrightarrow V_{\mathbb{P}^{1}}^{*} \mathcal{H}_{\bar{d}}
$$

are vector bundle inclusions. The boundary of $\mathcal{H} \mathcal{Q}_{\bar{d}}$, by which we mean the complement of $H_{\bar{d}}$, is therefore the locus $\mathcal{B}_{\bar{d}}$ such that on $\mathbb{P}^{1} \times \mathcal{B}_{\bar{d}}$ some of these maps degenerate. In this section we will study the restrictions of the generalized Schubert varieties $\bar{\Omega}_{w}(t)$ to $\mathcal{B}_{\bar{d}}$. We start with a description of $\mathcal{B}_{\bar{d}}$ itself, for which the following construction (taken from [C-F2, 2.2]) is needed:

Let $\bar{e}=\left(e_{1}, \ldots, e_{k}\right)$ be a multiindex satisfying the conditions

(5.1) $e_{i} \leq \min \left(n_{i}, d_{i}\right)$, for $1 \leq i \leq k$,

(5.2) $e_{i}-e_{i-1} \leq n_{i}-n_{i-1}$, for $2 \leq i \leq k$.

(cf. [C-F2, Lemma 2.1]).

For each $1 \leq i \leq k$, let $\pi_{i}: \mathcal{G}_{i} \longrightarrow \mathbb{P}^{1} \times \mathcal{H}_{\bar{d}-\bar{e}}$ be the Grassmann bundle of $e_{i}$-dimensional quotients of $\mathcal{S}_{i}^{\bar{d}-\bar{e}}$. Let $\mathcal{G}_{\bar{e}}$ be the fiber product of the $\mathcal{G}_{i}$ 's over $\mathbb{P}^{1} \times \mathcal{H} \mathcal{Q}_{\bar{d}-\bar{e}}$, with projection $\pi: \mathcal{G}_{\bar{e}} \longrightarrow \mathbb{P}^{1} \times \mathcal{H} \mathcal{Q}_{\bar{d}-\bar{e}}$.

For each $1 \leq i \leq k$, let

$$
0 \longrightarrow K_{i} \longrightarrow \pi_{i}^{*} \mathcal{S}_{i}^{\bar{d}-\bar{e}} \longrightarrow L_{i} \longrightarrow 0
$$

be the universal sequence on $\mathcal{G}_{i} . K_{i}$ and $L_{i}$ are vector bundles, of ranks $n_{i}-e_{i}$ and $e_{i}$ respectively. On $\mathcal{G}_{\bar{e}}$ we have the following diagram:

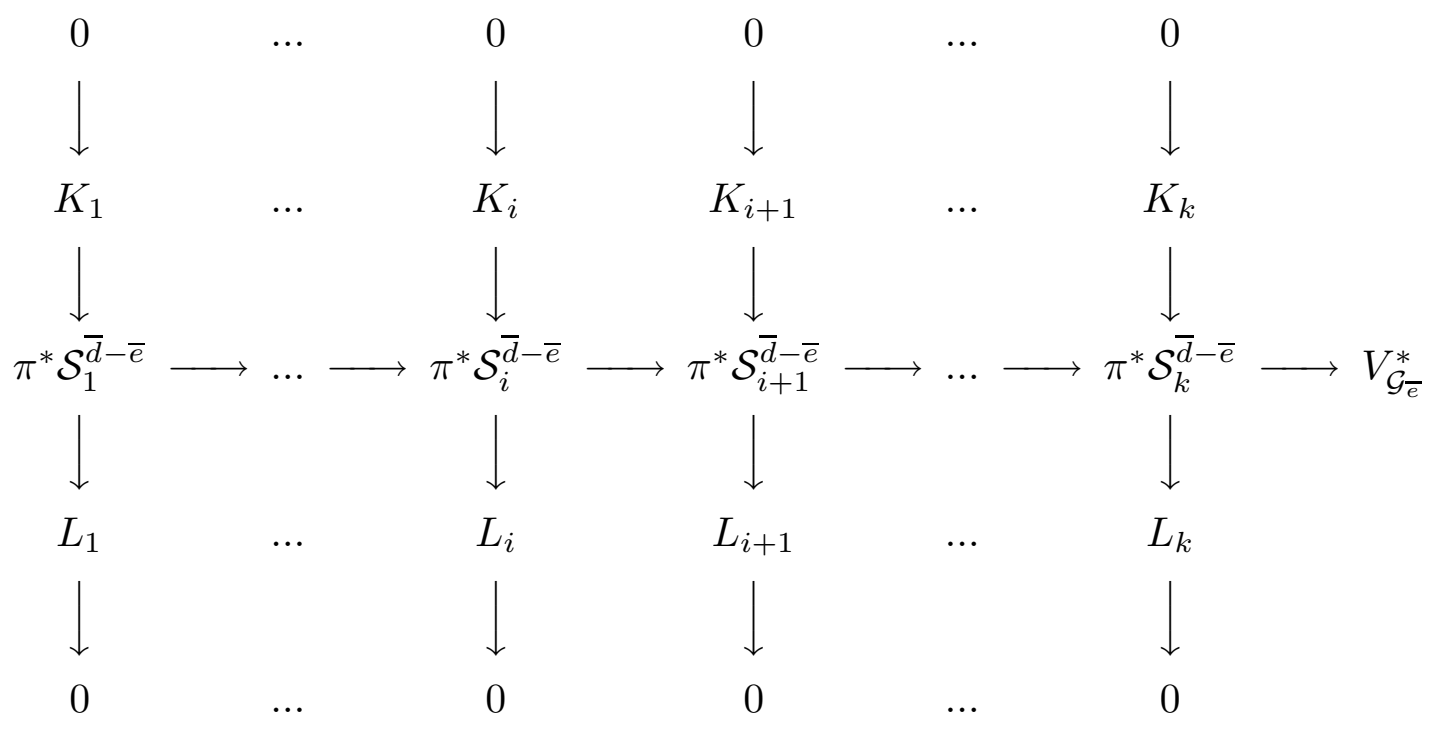

Let $\mathcal{U}_{\bar{e}}$ be the locally-closed subscheme of $\mathcal{G}_{\bar{e}}$ determined by the closed conditions

$$
\operatorname{rank}\left(K_{i} \longrightarrow L_{i+1}\right)=0, \quad \text { for } i=1, \ldots, k-1,
$$

and the open conditions

$$
\operatorname{rank}\left(K_{i} \longrightarrow V_{\mathcal{G}_{\bar{e}}}^{*}\right)=n_{i}-e_{i}, \quad \text { for } i=1, \ldots, k
$$


Lemma 5.1. $\mathcal{U}_{\bar{e}}$ is smooth, irreducible, of dimension

$$
1+\operatorname{dim}\left(\mathcal{H} \mathcal{Q}_{\bar{d}}\right)-\sum_{i=1}^{k} e_{i}\left(n_{i+1}-n_{i}\right)-\sum_{i=1}^{k} e_{i}\left(e_{i}-e_{i-1}\right)
$$

The projection $\pi: \mathcal{U}_{\bar{e}} \rightarrow \mathbb{P}^{1} \times \mathcal{H} \mathcal{Q}_{\bar{d}-\bar{e}}$ is smooth, and its image contains $\mathbb{P}^{1} \times H_{\bar{d}-\bar{e}}$.

Proof. For a vector bundle $E$ on a scheme $X$, we denote by $G_{e}(E)$ the Grassmann bundle of $e$-dimensional quotients of $E$, for some $0 \leq e \leq \operatorname{rank}(E)$.

Let $\mathcal{V}_{\bar{e}} \subset \mathcal{G}_{\bar{e}}$ be the open subscheme defined by the conditions (5.4) and put $\mathcal{V}:=\pi\left(\mathcal{V}_{\bar{e}}\right)$. Obviously, $\mathcal{V}$ is open in $\mathbb{P}^{1} \times \mathcal{H} \mathcal{Q}_{\bar{d}-\bar{e}}$, and contains $\mathbb{P}^{1} \times H_{\bar{d}-\bar{e}}$. The Lemma is a consequence of the observation that $\mathcal{U}_{\bar{e}}$ can be constructed as a sequence of $k$ Grassmann bundles over $\mathcal{V}$ as follows:

- start with $\rho_{1}: G_{e_{1}}\left(\mathcal{S}_{1}^{\bar{d}-\bar{e}}\right) \rightarrow \mathcal{V}$ with universal subbundle $K_{1}$;

- next, form $\rho_{2}: G_{e_{2}}\left(\rho_{1}^{*} \mathcal{S}_{2}^{\bar{d}-\bar{e}} / K_{1}\right) \rightarrow G_{e_{1}}\left(\mathcal{S}_{1}^{\bar{d}-\bar{e}}\right)$ with universal subbundle $\mathcal{L}_{2}$, and let $K_{2}$ be the natural induced extension

$$
0 \rightarrow \rho_{2}^{*} K_{1} \rightarrow K_{2} \rightarrow \mathcal{L}_{2} \rightarrow 0
$$

- continue by forming $\rho_{3}: G_{e_{3}}\left(\rho_{2}^{*} \rho_{1}^{*} \mathcal{S}_{3}^{\bar{d}-\bar{e}} / K_{2}\right) \rightarrow G_{e_{2}}\left(\rho_{1}^{*} \mathcal{S}_{2}^{\bar{d}-\bar{e}} / K_{1}\right)$, with universal subbundle $\mathcal{L}_{3}$, and let $K_{3}$ be the natural extension

$$
0 \rightarrow \rho_{3}^{*} K_{2} \rightarrow K_{3} \rightarrow \mathcal{L}_{3} \rightarrow 0
$$

and so on.

We will use this description of $\mathcal{U}_{\bar{e}}$ in the proof of Quantum Pieri (see 6.3).

Theorem 5.2. There exist morphisms $\phi_{\bar{e}}: \mathcal{U}_{\bar{e}} \longrightarrow \mathcal{H} \mathcal{Q}_{\bar{d}}$ such that

(i) If $\operatorname{rank}_{(t, x)} \mathcal{T}_{k-i+1}^{\bar{d}}=n-n_{i}+e_{i}$ for every $1 \leq i \leq k$ at a point $(t, x) \in$ $\mathbb{P}^{1} \times \mathcal{H} \mathcal{Q}_{\bar{d}}$, then $x \in \phi_{\bar{e}}\left(\mathcal{U}_{\bar{e}}\right)$. In particular $\mathcal{B}_{\bar{d}}$ is covered by the union of $\phi_{\bar{e}}\left(\mathcal{U}_{\bar{e}}\right)$, where $\bar{e}$ ranges over all (nonzero) multiindices satisfying (5.1) and (5.2).

(ii) The restriction of $\phi_{\bar{e}}$ to $\pi^{-1}\left(\mathbb{P}^{1} \times H_{\bar{d}-\bar{e}}\right)$ is an isomorphism onto its image.

Proof. See [C-F2, Theorem $2.3(i i)]$.

\section{Lemma 5.3.}

$$
\phi_{\bar{e}}^{-1}\left(\bar{\Omega}_{w}(t)\right)=\pi^{-1}\left(\mathbb{P}^{1} \times \bar{\Omega}_{w}(t)\right) \bigcup \tilde{\Omega}_{w}^{\bar{e}}(t)
$$

$\tilde{\Omega}_{w}^{\bar{e}}(t)$ being the locus inside $\mathcal{U}_{\bar{e}}(t):=\pi^{-1}\left(\{t\} \times \mathcal{H} \mathcal{Q}_{\bar{d}-\bar{e}}\right)$ where

$$
\operatorname{rank}\left(V_{p} \otimes \mathcal{O} \rightarrow K_{q}^{*}\right) \leq r_{w}(q, p)
$$

for all $p=1, \ldots, n, q \in\left\{n_{1}, \ldots, n_{k}\right\}$.

Proof. See [C-F2, Lemma 3.1].

Following [C-F2, Section 3] we will describe now the locus $\tilde{\Omega}_{w}^{\bar{e}}(t)$ of Lemma 5.3. The analysis there can be applied in our case without any changes and the only reason for reproducing part of it here is to fix the somewhat elaborate notation 
Let $a:=\operatorname{card}\left\{n_{i}-e_{i} \mid i=1, \ldots, k\right\}$. Set $e_{0}=e_{k+1}=0$ and define a partition of $[0, k+1]$ as follows:

$$
i_{0}=0, i_{j}=\min \left\{i \mid n_{i}-e_{i} \geq n_{i_{j-1}}-e_{i_{j-1}}+1\right\}, \text { for } 1 \leq j \leq a, i_{a+1}=k+1 .
$$

Let $m_{j}=n_{i_{j}}-e_{i_{j}}$, for $j=0,1, \ldots, a$. By definition, on each of the intervals

$$
\left[1, i_{1}-1\right],\left[i_{1}, i_{2}-1\right], \ldots,\left[i_{a-1}, i_{a}-1\right],\left[i_{a}, k\right]
$$

$n_{i}-e_{i}$ is constant, equal respectively to $0, m_{1}, \ldots, m_{a}$, and the corresponding bundles $K_{i}^{*}$ are all isomorphic. Therefore we can restrict the set of rank conditions (5.5), defining $\tilde{\Omega}_{w}^{\bar{e}}(t)$ inside $\mathcal{U}_{\bar{e}}(t)$, to

$$
\operatorname{rank}\left(V_{p} \otimes \mathcal{O} \rightarrow K_{q}^{*}\right) \leq r_{w}(q, p), 1 \leq p \leq n, q \in\left\{n_{i_{1}}, \ldots, n_{i_{a}}\right\} .
$$

Moreover, we can further modify (5.6). Define recursively $\mathbf{r}:=\left(r_{j, p}\right)_{1 \leq p \leq n, 1 \leq j \leq a}$ as follows:

$$
\begin{gathered}
r_{1, p}=\min \left\{r_{w}\left(n_{i_{1}}, p\right), m_{1}\right\}, 1 \leq p \leq n, \\
r_{j, p}=\min \left\{r_{w}\left(n_{i_{j}}, p\right), r_{j-1, p}+m_{j}-m_{j-1}\right\}, 1 \leq p \leq n, 2 \leq j \leq a .
\end{gathered}
$$

Lemma 5.4. The conditions

$$
\operatorname{rank}\left(V_{p} \otimes \mathcal{O} \rightarrow K_{i_{j}}^{*}\right) \leq r_{j, p}, 1 \leq p \leq n, 1 \leq j \leq a
$$

define the same degeneracy locus $\tilde{\Omega}_{w}^{\bar{e}}(t)$ in $\mathcal{U}_{\bar{e}}(t)$.

Proof. See [C-F2, 3.5].

Lemma 5.5. (i) There exists a (unique) permutation $\tilde{w}^{\bar{e}} \in S_{n}$ such that if $\tilde{w}^{\bar{e}}(q)>$ $\tilde{w}^{\bar{e}}(q+1)$, then $q \in\left\{m_{1}, \ldots, m_{a}\right\}$, and $r_{j, p}=r_{\tilde{w}^{e}}\left(m_{j}, p\right)$, for all $1 \leq p \leq n, 1 \leq$ $j \leq a$.

(ii) $\ell(w)-\ell\left(\tilde{w}^{\bar{e}}\right) \leq \sum_{i=1}^{k} e_{i}\left(n_{i+1}-n_{i}\right)$.

Proof. The following explicit construction of $\tilde{w}^{\bar{e}}$ is taken from [C-F2, Lemma 3.6].

For each $j=0,1, \ldots, a+1$, define sets $W_{j}(w)$, by

$$
W_{0}(w)=\emptyset, W_{j}(w)=\left\{w(1), \ldots, w\left(n_{i_{j}}\right)\right\} .
$$

Also we define sets $Z_{j}(w)$, and ordered sets $\tilde{Z}_{j}(w)$, such that

(a) $\operatorname{card} Z_{j}(w)=n_{i_{j}}-m_{j-1}$,

(b) $\operatorname{card} \tilde{Z}_{j}(w)=m_{j}-m_{j-1}$,

(c) $\tilde{Z}_{j}(w) \cap \tilde{Z}_{j^{\prime}}(w)=\emptyset$ if $j \neq j^{\prime}$,

(d) $\bigcup_{j=1}^{a+1} \tilde{Z}_{j}(w)=[1, n]$,

by the following recursive procedure:

Let $Z_{0}(w)=\tilde{Z}_{0}(w)=\emptyset$. If $\tilde{Z}_{i}(w)$ has been already defined for $i=0,1, \ldots, j-1$, let

$$
Z_{j}(w)=W_{j}(w) \backslash\left(\bigcup^{j-1} \tilde{Z}_{i}(w)\right)
$$


Arrange $Z_{j}(w)$ in increasing order

$$
Z_{j}(w)=\left\{z_{j, 1}<\cdots<z_{j, n_{i_{j}}-m_{j-1}}\right\}
$$

and set

$$
\begin{gathered}
\tilde{z}_{m_{j-1}+1}:=z_{j, 1}, \tilde{z}_{m_{j-1}+2}:=z_{j, 2}, \ldots, \tilde{z}_{m_{j}}:=z_{j, m_{j}-m_{j-1}}, \\
\tilde{Z}_{j}(w):=\left\{\tilde{z}_{m_{j-1}+1}<\tilde{z}_{m_{j-1}+2}<\cdots<\tilde{z}_{m_{j}}\right\} .
\end{gathered}
$$

Now define $\tilde{w}^{\bar{e}}(q)=\tilde{z}_{q}$, for all $1 \leq q \leq n$.

The estimate (ii) follows (cf. [C-F2, Lemma 3.8]) by noticing first that the difference $\ell(w)-\ell\left(\tilde{w}^{\bar{e}}\right)$ is maximized by the longest permutation $w^{\circ}$, defined by $w^{\circ}(i)=n-n_{j}+i-n_{j-1}$, for all $n_{j-1}+1 \leq i \leq n_{j}, 1 \leq j \leq k+1$. For this case one computes directly that we have in fact the equality

$$
\ell\left(w^{\circ}\right)-\ell\left(\left(\tilde{w}^{\circ}\right)^{\bar{e}}\right)=\sum_{i=1}^{k} e_{i}\left(n_{i+1}-n_{i}\right) .
$$

Remark 5.6. ( $i)$ In the terminology of [F1], Lemma $5.5(i)$ says that $\mathbf{r}$ is a permissible collection of rank numbers.

(ii) Let $\tilde{F}_{\bar{e}}:=F\left(m_{1}, \ldots, m_{a}, V\right)$ be the partial flag variety corresponding to the $m_{i}$ 's. The sequence of quotient bundles

$$
V \otimes \mathcal{O}_{\mathcal{U}_{\bar{e}}(t)} \rightarrow K_{n_{i_{a}}}^{*} \rightarrow \cdots \rightarrow K_{n_{i_{1}}}^{*}
$$

is the pull-back via an uniquely determined morphism $\psi_{\bar{e}}(t): \mathcal{U}_{\bar{e}}(t) \rightarrow \tilde{F}_{\bar{e}}$ of the tautological sequence on $\tilde{F}_{\bar{e}}$. By Lemmas 5.4 and $5.5(i), \tilde{w}^{\bar{e}}$ defines a Schubert variety on $\tilde{F}_{\bar{e}}$, and we have $\tilde{\Omega}_{w}^{\bar{e}}(t)=\psi_{\bar{e}}(t)^{-1}\left(\Omega_{\left.\tilde{w}^{\bar{e}}\right)}\right.$.

Finally, we spell out in more detail what the analysis in this section says for some special cases.

Lemma 5.7. Let $\left(e_{1}, \ldots, e_{k}\right)$ be a multiindex. Fix $1 \leq j \leq k$ and $1 \leq i \leq n_{j}$ and consider the cycle $\alpha_{i, j}:=s_{n_{j}-i+1} \cdot \ldots \cdot s_{n_{j}}$. Then

$$
\tilde{\alpha}_{i, j}^{\bar{e}}=\left\{\begin{array}{l}
\alpha_{i, j}, \quad \text { if } e_{j}=0 \\
\alpha_{i, j} \cdot s_{n_{j}} \cdot \ldots \cdot s_{n_{j}-e_{j}+1}, \quad \text { if } 1 \leq e_{j}<i \\
i d, \quad \text { if } i \leq e_{j} .
\end{array}\right.
$$

In particular, $\ell\left(\alpha_{i, j}\right)-\ell\left(\tilde{\alpha}_{i, j}^{\bar{e}}\right) \leq e_{j}$, with equality iff $e_{j} \leq i$.

Proof. Immediate from the construction of $\tilde{\alpha}_{i, j}^{\bar{e}}$ in Lemma 5.5.

Lemma 5.8 (cf. [C-F2, Lemma 3.9]). Assume in addition that $\bar{e} \neq(0 \ldots, 0)$. Then

(i) $\sum_{i=1}^{k} e_{i}\left(e_{i}-e_{i-1}\right) \geq e_{j}$, for $1 \leq j \leq k$. In particular $\sum_{i=1}^{k} e_{i}\left(e_{i}-e_{i-1}\right) \geq 1$.

(ii) There exists $1 \leq j \leq k$ such that $\sum_{i=1}^{k} e_{i}\left(e_{i}-e_{i-1}\right)=e_{j}$ iff the following 
there are integers $1 \leq h_{1}<h_{2}<\cdots<h_{m} \leq j \leq l_{m}<\cdots<l_{2}<l_{1} \leq k$ such that

$$
e_{i}=\left\{\begin{array}{l}
0, \text { for } i \in\left[1, h_{1}-1\right] \cup\left[l_{1}+1, k\right], \\
1, \text { for } i \in\left[h_{1}, h_{2}-1\right] \cup\left[l_{2}+1, l_{1}\right], \\
2, \text { for } i \in\left[h_{2}, h_{3}-1\right] \cup\left[l_{3}+1, l_{2}\right], \\
\cdots \\
m, \text { for } i \in\left[h_{m}, l_{m}\right]
\end{array}\right.
$$

(in particular, $e_{j}=m$ ).

(iii) Let $\bar{e}_{\mathbf{h l}}$ denote a multiindex as in $($ ii), and let $w \in S$ be any permutation. Let $\tilde{w}^{\bar{e}_{\mathbf{h}}}$ be the permutation given by Lemma $5.5(i)$. Then

$$
\ell(w)-\ell\left(\tilde{w}^{\bar{e}_{\mathbf{h l}}}\right)=\sum_{i=1}^{k} e_{i}\left(n_{i+1}-n_{i}\right)=\sum_{c=1}^{m}\left(n_{l_{c}+1}-n_{h_{c}}\right)
$$

iff for every $1 \leq i \leq m$ we have

$$
w\left(n_{h_{i}}\right)>\max \left\{w\left(n_{h_{i}}+1\right), \ldots, w\left(n_{l_{i}}\right), w\left(n_{l_{i}+1}\right)\right\}
$$

In this case, $\tilde{w}^{\bar{e}_{\mathbf{h l}}}=w \cdot \gamma_{h_{m}, l_{m}} \cdot \gamma_{h_{m-1}, l_{m-1}} \cdot \ldots \cdot \gamma_{h_{1}, l_{1}}$, where $\gamma_{h, l}$ denotes the cyclic permutation $s_{n_{h}} \cdot \ldots \cdot s_{n_{l+1}-1}$ (cf. the paragraph before Theorem 3.1).

Proof. (i) First, using the easy identity

$$
\sum_{i=1}^{k} e_{i}\left(e_{i}-e_{i-1}\right)=\frac{1}{2}\left[e_{1}^{2}+\left(e_{2}-e_{1}\right)^{2}+\cdots+\left(e_{k}-e_{k-1}\right)^{2}+e_{k}^{2}\right]
$$

and the change of variables

$$
x_{1}=e_{1}, x_{2}=e_{2}-e_{1}, \ldots, x_{k}=e_{k}-e_{k-1}, x_{k+1}=e_{k},
$$

the inequality in $(i)$ becomes

$$
\sum_{i=1}^{j}\left(x_{i}^{2}-2 x_{i}\right)+\sum_{i=j+1}^{k+1} x_{i}^{2} \geq 0
$$

with the additional constraint $x_{k+1}=\sum_{i=1}^{k} x_{i}$. Now (5.8) is equivalent to

$$
\sum_{i=1}^{j}\left(x_{i}-1\right)^{2}+\sum_{i=j+1}^{k+1} x_{i}^{2} \geq j
$$

Making another change of variables

$$
y_{1}=x_{1}-1, \ldots, y_{j}=x_{j}-1, y_{j+1}=x_{j+1}, \ldots, y_{k+1}=x_{k+1},
$$

we are reduced to proving

$$
\sum^{k+1} y_{i}^{2} \geq j
$$


subject to the constraint

$$
y_{k+1}=j+\sum_{i=1}^{k} y_{i}
$$

Replacing in (5.10) $j$ by $y_{k+1}-\sum_{i=1}^{k} y_{i}$, we get

$$
\left(y_{k+1}^{2}-y_{k+1}\right)+\sum_{i=1}^{k}\left(y_{i}^{2}+y_{i}\right) \geq 0 \text {. }
$$

Since $y_{i}, 1 \leq i \leq k+1$ are integers, each paranthesis in (5.12) is nonnegative. This proves $(i)$.

(ii) We have equality in (5.12) iff $y_{k+1}$ is equal to either 0 or 1 , and each $y_{i}, 1 \leq$ $i \leq k$ is equal to either 0 or -1 . Using (5.11), we see that equality occurs in one of the following two cases

- either $y_{k+1}=0$, exactly $j$ among $y_{1} \ldots, y_{k}$ are equal to -1 , and the rest of them are equal to 0 ,

- or $y_{k+1}=1$, exactly $j-1$ among $y_{1} \ldots, y_{k}$ are equal to -1 , and the rest of them are equal to 0.

Changing the variables back to $e_{i}$, the statement in $(i i)$ is obtained.

(iii) follows by the construction of $\tilde{w}^{\bar{e}_{\mathbf{h} 1}}$.

\section{Proofs of the Moving Lemma, the Quantum \\ Pieri Formula, And the Orthogonality Theorem}

This section is devoted to the proofs of Theorem 4.4 (ii), Theorem 3.1, and Theorem 3.16. For this purpose we will use heavily the structure of the boundary of $\mathcal{H} \mathcal{Q}_{\bar{d}}$, described in the preceding section.

Throughout the rest of the paper, we will work with suitable general translates of the Schubert varieties on $F$.

\subsection{Proof of Theorem 4.4 (ii) (cf. [C-F2, Theorem 4.1]).}

We proceed by induction on $\bar{d}$. If $d_{1}=\cdots=d_{k}=0$, then $\mathcal{H} \mathcal{Q}_{\bar{d}}=H_{\bar{d}}=F$ and there is nothing to prove. Assume that the statement is true for all $\bar{f}$ such that $f_{i} \leq d_{i}, 1 \leq i \leq k$ and $f_{j}<d_{j}$ for some $1 \leq j \leq k$. Let $c:=\sum_{i=1}^{N} \ell\left(w_{i}\right)$. By $(i)$ and Theorem $5.2(i)$, it is enough to show that

$$
\operatorname{codim}_{\mathcal{H} \mathcal{Q}_{\bar{d}}}\left(\bigcap_{i=1}^{N} \bar{\Omega}_{w_{i}}\left(t_{i}\right)\right) \bigcap \phi_{\bar{e}}\left(\mathcal{U}_{\bar{e}}\right)>c
$$

for every multiindex $\bar{e} \neq(0, \ldots, 0)$, satisfying the conditions (5.1) and (5.2). Using Theorem 5.2 (ii) and Lemma 5.1, the inequality (6.1) will follow if we prove that the codimension of $\bigcap_{i=1}^{N} \phi_{\bar{e}}^{-1}\left(\bar{\Omega}_{w_{i}}\left(t_{i}\right)\right)$ in $\mathcal{U}_{\bar{e}}$ is greater than

$$
c-\left(\operatorname{dim} \mathcal{H} \mathcal{Q}_{\bar{d}}-\operatorname{dim} \mathcal{U}_{\bar{e}}\right)=c+1-\sum^{k} e_{i}\left(n_{i+1}-n_{i}\right)-\sum^{k} e_{i}\left(e_{i}-e_{i-1}\right)
$$


By Lemma 5.3, we have to prove the same estimate for the codimension of

$$
\bigcap_{i=1}^{N}\left(\pi^{-1}\left(\mathbb{P}^{1} \times \bar{\Omega}_{w_{i}}\left(t_{i}\right)\right) \bigcup \tilde{\Omega}_{w_{i}}^{\bar{e}}\left(t_{i}\right)\right)
$$

in $\mathcal{U}_{\bar{e}}$. Since the points $t_{1}, \ldots, t_{N}$ are distinct, the only possibly nonempty intersections in (6.2) contain either no $\tilde{\Omega}_{w_{i}}^{\bar{e}}\left(t_{i}\right)$ term, or only one such term. If there is no such term, the required inequality follows from the induction assumption on $\mathcal{H} \mathcal{Q}_{\bar{d}-\bar{e}}$ and the fact that $\pi$ is a smooth map. After possibly renumbering the points $t_{i}$, to finish the proof it suffices to show the estimate for

$$
W \bigcap \tilde{\Omega}_{w_{N}}^{\bar{e}}\left(t_{N}\right) \subset \mathcal{U}_{\bar{e}}\left(t_{N}\right),
$$

where

$$
W:=\bigcap_{i=1}^{N-1}\left(\pi^{-1}\left(\left\{t_{N}\right\} \times \bar{\Omega}_{w_{i}}\left(t_{i}\right)\right)\right),
$$

and $\mathcal{U}_{\bar{e}}\left(t_{N}\right)=\pi^{-1}\left(\left\{t_{N}\right\} \times \mathcal{H} \mathcal{Q}_{\bar{d}-\bar{e}}\right)$. By the induction assumption, $\mathrm{W}$ has codimension $c-\ell\left(w_{N}\right)$ in $\mathcal{U}_{\bar{e}}\left(t_{N}\right)$, while by Remark 5.6 (ii) and Kleiman's Theorem on transversality of general translates, the intersection (6.3) has codimension $\ell\left(\tilde{w}^{\bar{e}}\right)$ in $W$. The estimate follows now from Lemma $5.5(i i)$ and Lemma $5.8(i)$.

\subsection{Computing GW-invariants via degenerations.}

For the proofs of Quantum Pieri and Orthogonality, we need to compute certain invariants $\left\langle\Omega_{w_{1}}, \ldots, \Omega_{w_{N}}\right\rangle_{\bar{d}}$. The technique we will use is to degenerate the intersection $\bigcap_{i=1}^{N} \bar{\Omega}_{w_{i}}\left(t_{i}\right)$ by allowing some of the points $t_{i}$ to coincide. This procedure may lead to contributions supported on the boundary, which can be evaluated using the analysis in Section 5. In this subsection we summarize some results of this type.

The following is an easy consequence of Proposition 4.9 and Theorem 4.4. For a proof, see for instance [Be, Lemma 2.5].

Lemma 6.1. Let $Y_{1}, Y_{2}$ be subvarieties in $F$ such that $\operatorname{codim} Y_{1}+\operatorname{codim} Y_{1}=$ $\operatorname{dim} \mathcal{H} \mathcal{Q}_{\bar{d}}$, and let $t_{1}, t_{2} \in \mathbb{P}^{1}$ be distinct points. Assume $\bar{d} \neq(0, \ldots, 0)$. Then

$$
\int_{\mathcal{H}_{\bar{d}}}\left[\overline{Y_{1}\left(t_{1}\right)}\right] \cup\left[\overline{Y_{2}\left(t_{2}\right)}\right]=0
$$

In particular, for any $v, w \in S$,

$$
\left\langle\Omega_{v}, \Omega_{w}\right\rangle_{\bar{d}}=\left\{\begin{array}{ll}
1, & \text { if } \bar{d}=(0, \ldots, 0) \text { and } v=\check{w} \\
0, & \text { otherwise }
\end{array} .\right.
$$

Lemma 6.2. Let $\bar{d}$ be a multiindex, and let $v_{1}, \ldots, v_{N}, w_{1}, \ldots, w_{M} \in S$ satisfy $\sum_{i=1}^{N} \ell\left(v_{i}\right)+\sum_{j=1}^{M} \ell\left(w_{j}\right)=\operatorname{dim} H_{\bar{d}}$. Let $y, t_{1}, \ldots, t_{M} \in \mathbb{P}^{1}$ be distinct points. Assume that the conclusion of Theorem 4.4 (ii) holds for the intersection 
Let $Y:=\Omega_{v_{1}} \cap \cdots \bigcap \Omega_{v_{N}} \subset F$. Then

$$
\left\langle\Omega_{v_{1}}, \ldots, \Omega_{v_{N}}, \Omega_{w_{1}}, \ldots, \Omega_{w_{M}}\right\rangle_{\bar{d}}=\int_{\mathcal{H}_{\bar{Q}}}[\overline{Y(y)}] \cup\left[\bar{\Omega}_{w_{1}}\left(t_{1}\right)\right] \cup \cdots \cup\left[\bar{\Omega}_{w_{N}}\left(t_{N}\right)\right]
$$

Proof. By Theorem $4.4(i)$, the intersection

$$
\Omega_{v_{1}}(y) \bigcap \cdots \bigcap \Omega_{v_{N}}(y) \bigcap \Omega_{w_{1}}\left(t_{1}\right) \bigcap \cdots \bigcap \Omega_{w_{M}}\left(t_{M}\right) \subset H_{\bar{d}}
$$

has pure dimension 0, and by assumption it coincides with (6.4). On the other hand, we have

$$
\Omega_{v_{1}}(y) \bigcap \cdots \bigcap \Omega_{v_{N}}(y)=e v^{-1}(Y) \bigcap\{y\} \times H_{\bar{d}}
$$

The Lemma follows now from the definition of $\langle\ldots\rangle_{\bar{d}}$, Corollaries 4.5 and 4.6 , and Proposition 4.9.

Proposition 6.3. Let $\bar{d} \neq(0, \ldots, 0)$ be a multiindex, let $u, v, w \in S$ be such that $\ell(u)+\ell(v)+\ell(w)=\operatorname{dim} H_{\bar{d}}$, and let $y, t \in \mathbb{P}^{1}$ be distinct points. Denote

$$
Z:=\bar{\Omega}_{u}(y) \bigcap \bar{\Omega}_{v}(y) \bigcap \bar{\Omega}_{w}(t) \subset \mathcal{H} \mathcal{Q}_{\bar{d}}
$$

Assume that $Z$ is either empty, or purely 0-dimensional. Then

(i) $Z$ is contained in $\mathcal{B}_{\bar{d}}$.

(ii) $[Z]=\left[\bar{\Omega}_{u}(y)\right] \cup\left[\bar{\Omega}_{v}(y)\right] \cup\left[\bar{\Omega}_{w}(t)\right]$ is a cycle of degree $\left\langle\Omega_{u}, \Omega_{v}, \Omega_{w}\right\rangle_{\bar{d}}$.

Proof. ( $i$ ) Write $\Omega_{u} \cap \Omega_{v}=Y$ inside $F$. Let $Z^{\prime}$ be the (largest) subscheme of $Z$ supported on $H_{\bar{d}}$. Then, as in the proof of Lemma 6.2, we have

$$
Z^{\prime}=Y(y) \bigcap \Omega_{w}(t)
$$

hence, by Proposition 4.9,

$$
\operatorname{card} Z^{\prime}=\int_{\mathcal{H}_{\bar{d}}}[\overline{Y(y)}] \cup\left[\bar{\Omega}_{w}(t)\right]
$$

By Lemma 6.1, $Z^{\prime}$ is empty.

(ii) Let $V:=\bar{\Omega}_{v}(y) \cap \bar{\Omega}_{w}(t) \subset \mathcal{H} \mathcal{Q}_{\bar{d}}$, and consider the trivial family $\mathbb{P}^{1} \times V \subset$ $\mathbb{P}^{1} \times \mathcal{H} \mathcal{Q}_{\bar{d}}$ over $\mathbb{P}^{1}$. Let $\rho: X \hookrightarrow \mathbb{P}^{1} \times \mathcal{H} \mathcal{Q}_{\bar{d}} \stackrel{p r}{\longrightarrow} \mathbb{P}^{1}$ be the family whose fibre over $x \in \mathbb{P}^{1}$ is the generalized Schubert variety $\bar{\Omega}_{u}(x)$. It follows from Theorem 4.4 that $\rho$ is a fibre bundle map (see e.g. [Be, Corollary 2.4]), and in particular it is flat. Since the intersection $\left(\mathbb{P}^{1} \times V\right) \bigcap X$ is obviously proper over $\mathbb{P}^{1}$, the Proposition follows from Example 10.2.1 in [F2]. 


\subsection{Proof of Quantum Pieri.}

We formulate first an auxiliary Lemma, for which we introduce some notation.

Let $X$ be a scheme. Let $V$ be an $n$-dimensional complex vector space and let $B_{i}$, $1 \leq i \leq k$ be vector bundles on $X$, of ranks $b_{i}$ respectively. Fix $1 \leq j \leq k$. Assume that we are given a sequence of generically injective maps

$$
B_{1} \rightarrow \cdots \rightarrow B_{j-1} \rightarrow B_{j} \rightarrow B_{j+1} \rightarrow \cdots \rightarrow B_{k} \rightarrow V^{*} \otimes \mathcal{O}_{X}
$$

Moreover, assume that $B_{i} \rightarrow V^{*}$ is an injective map of bundles for $1 \leq i \leq j$. Fix $0 \leq e \leq b_{j}-b_{j-1}$, and let $\rho: G_{e}\left(B_{j} / B_{j-1}\right) \rightarrow X$ be the Grassmann bundle of $e$-dimensional quotients of $B_{j} / B_{j-1}$, with universal sequence

$$
0 \rightarrow L \rightarrow \rho^{*}\left(B_{j} / B_{j-1}\right) \rightarrow Q \rightarrow 0
$$

Let $K$ be the natural induced extension

$$
0 \rightarrow \rho^{*} B_{j-1} \rightarrow K \rightarrow L \rightarrow 0
$$

i.e., $K$ is the kernel of $\rho^{*} B_{j} \rightarrow Q$.

Let $V_{1} \subset \cdots \subset V_{n-1} \subset V$ be a fixed flag, and let $w \in S_{n}$ be a permutation such that if $w(q)>w(q+1)$, then $q \in\left\{b_{1}, \ldots, b_{j-1}, b_{j}-e, b_{j+1}, \ldots, b_{k}\right\}$. Denote by $\mathbf{D}_{w}$ the degeneracy locus on $G_{e}\left(B_{j} / B_{j-1}\right)$ determined by

$\operatorname{rank}\left(V_{p} \otimes \mathcal{O} \rightarrow\left(\rho^{*} B_{q}\right)^{*}\right) \leq r_{w}(q, p), 1 \leq p \leq n, q \in\left\{b_{1}, \ldots, b_{j-1}, b_{j+1}, \ldots, b_{k}\right\}$,

and

$$
\operatorname{rank}\left(V_{p} \otimes \mathcal{O} \rightarrow K^{*}\right) \leq r_{w}\left(b_{j}-e, p\right), 1 \leq p \leq n
$$

Define a permutation $\hat{w} \in S_{n}$ as follows:

- let $\left\{z_{1}<z_{2}<\cdots<z_{b_{j}-b_{j-1}}\right\}$ be the set $\left\{w\left(b_{j-1}+1\right), w\left(b_{j-1}+2\right), \ldots, w\left(b_{j}\right)\right\}$, ordered increasingly;

- if $q \notin\left\{b_{j-1}+1, b_{j-1}+2, \ldots, b_{j}\right\}$, set $\hat{w}(q)=w(q)$;

- for $1 \leq i \leq b_{j}-b_{j-1}$, set $\hat{w}\left(b_{j-1}+i\right)=z_{i}$.

Lemma 6.4. (i) The image $\rho\left(\mathbf{D}_{w}\right)$ is the degeneracy locus $\mathbf{D}_{\hat{w}}$ on $X$ defined by

$$
\operatorname{rank}\left(V_{p} \otimes \mathcal{O} \rightarrow B_{q}^{*}\right) \leq r_{\hat{w}}(q, p), 1 \leq p \leq n, q \in\left\{b_{1}, \ldots, b_{k}\right\} .
$$

(ii) The restriction of $\rho$ to $\mathbf{D}_{w}$ has positive dimensional fibres, unless

$$
w\left(b_{j-1}+1\right)>w\left(b_{j}\right)
$$

in which case

$$
\hat{w}=w \cdot \underbrace{s_{b_{j}-e} \cdot \ldots \cdot s_{b_{j-1}+1}} \cdot \underbrace{s_{b_{j}-e+1} \cdot \ldots \cdot s_{b_{j-1}+2}} \cdot \cdots \cdot \underbrace{s_{b_{j}-1} \cdot \ldots \cdot s_{b_{j-1}+e}} .
$$

If (6.8) holds and $\mathbf{D}_{\hat{w}}$ is irreducible, then $\rho$ maps $\mathbf{D}_{w}$ birationally onto $\mathbf{D}_{\hat{w}}$.

Proof. Note first that if (6.8) is satisfied, then (6.9) follows directly from the defi- 
By the construction of $\hat{w}$, we have $r_{\hat{w}}\left(b_{j}, p\right)=r_{w}\left(b_{j}, p\right)$, for all $1 \leq p \leq n$. Since $w\left(b_{j}\right)<w\left(b_{j}+1\right)$ by assumption, it follows from [F1, Proposition 4.2] that by adding the conditions

$$
\operatorname{rank}\left(V_{p} \otimes \mathcal{O} \rightarrow\left(\rho^{*} B_{j}\right)^{*}\right) \leq r_{w}\left(b_{j}, p\right), 1 \leq p \leq n
$$

to (6.5) and (6.6), we obtain the same locus $\mathbf{D}_{w}$ on $G_{e}\left(B_{j} / B_{j-1}\right)$. In other words, $\mathbf{D}_{w}$ is contained in $\rho^{-1}\left(\mathbf{D}_{\hat{w}}\right)$. Consider the Grassmann bundle obtained by restriction

$$
\rho: \rho^{-1}\left(\mathbf{D}_{\hat{w}}\right) \rightarrow \mathbf{D}_{\hat{w}}
$$

It is not hard to see that $\mathbf{D}_{w}$ is a Schubert variety in this bundle, of positive relative dimension, unless (6.8) holds, in which case it intersects each fibre in a point, and the Lemma follows.

We will now prove the following equivalent reformulation of Theorem 3.1.

Theorem 3.1'. The $G W$-number $\left\langle\Omega_{\alpha_{i, j}}, \Omega_{w}, \Omega_{v}\right\rangle_{\bar{d}}$ vanishes, unless $\bar{d}$ is one of the multiindices $\bar{e}_{\mathbf{h l}}$ of Lemma 5.8, such that $\ell\left(w \cdot \gamma_{\mathbf{h l}}\right)=\ell(w)-\sum_{c=1}^{m}\left(n_{l_{c}+1}-n_{h_{c}}\right)$, and $v$ is dual to one of the permutations $w^{\prime \prime} \cdot \delta_{\mathbf{h l}}$, in which cases it is equal to 1 .

Proof of Theorem 3.1'. The idea is to degenerate the corresponding intersection of generalized Schubert varieties, as in the previous subsection.

Specifically, let $\bar{d}$ be any multiindex not identically 0 , and let $v \in S$ be such that $c:=i+\ell(w)+\ell(v)=\operatorname{dim} H_{\bar{d}}$. Let $y, t \in \mathbb{P}^{1}$ be distinct points.

We now claim that the intersection

$$
Z:=\bar{\Omega}_{\alpha_{i, j}}(y) \bigcap \bar{\Omega}_{w}(y) \bigcap \bar{\Omega}_{v}(t)
$$

is either empty, or purely 0-dimensional.

Indeed, by Theorem $4.4(i)$, it is enough to show that the restriction of $Z$ to $\mathcal{B}_{\bar{d}}$ is either empty, or purely of dimension 0. As in the proof of Theorem $4.4(\mathrm{ii})$, this reduces to showing that the codimension in $\mathcal{U}_{\bar{e}}$ of

$$
\left(\pi^{-1}\left(\mathbb{P}^{1} \times \bar{\Omega}_{\alpha_{i, j}}(y)\right) \bigcup \tilde{\Omega}_{\alpha_{i, j}}^{\bar{e}}(y)\right) \bigcap\left(\pi^{-1}\left(\mathbb{P}^{1} \times \bar{\Omega}_{w}(y)\right) \bigcup \tilde{\Omega}_{w}^{\bar{e}}(y)\right) \bigcap\left(\pi^{-1}\left(\mathbb{P}^{1} \times \bar{\Omega}_{v}(t)\right) \bigcup \tilde{\Omega}_{v}^{\bar{e}}(t)\right)
$$

is at least $c+1-\sum_{i=1}^{k} e_{i}\left(n_{i+1}-n_{i}\right)-\sum_{i=1}^{k} e_{i}\left(e_{i}-e_{i-1}\right)$, for all multiindices $\bar{e} \neq(0, \ldots, 0)$, satisfying $(5.1)$ and (5.2). We have seen already in the proof of Theorem 4.4 (ii) that the only intersection which may be nonempty is

$$
\tilde{\Omega}_{\alpha_{i, j}}^{\bar{e}}(y) \bigcap \tilde{\Omega}_{w}^{\bar{e}}(y) \bigcap \pi^{-1}\left(\{y\} \times \bar{\Omega}_{v}(t)\right),
$$

which lies inside $\mathcal{U}_{\bar{e}}(y)$. But we can rewrite (6.11) as

$$
\psi_{\bar{e}}(y)^{-1}\left(\Omega_{\tilde{\alpha}_{i, j}^{\bar{e}}}\right) \bigcap \psi_{\bar{e}}(y)^{-1}\left(\Omega_{\tilde{w}^{\bar{e}}}\right) \bigcap \pi^{-1}\left(\{y\} \times \bar{\Omega}_{v}(t)\right),
$$

where $\psi_{\bar{e}}(y): \mathcal{U}_{\bar{e}}(y) \rightarrow \tilde{F}_{\bar{e}}$ is the morphism of Remark 5.6 (ii). The codimension of (6.12) in $\mathcal{U}_{\bar{e}}(y)$ satisfies the required estimate by Kleiman's transversality Theorem, 
The conclusion of Proposition 6.3 applies therefore in this case, and the GWinvariant $\left\langle\Omega_{\alpha_{i, j}}, \Omega_{w}, \Omega_{v}\right\rangle_{\bar{d}}$ can be computed as the degree of $[Z]$ in the Chow ring of the hyperquot scheme. But we know even more! Namely, if $Z$ is nonempty, all the inequalities we have used above must be in fact equalities. By Lemma 5.8 ( $i i)$ and (iii), this implies that $Z$ is contained in the (disjoint!) union of "strata"

$$
\bigcup_{\bar{e}_{\mathbf{h} 1}} \phi_{\bar{e}_{\mathbf{h} 1}}\left(\mathcal{U}_{\bar{e}_{\mathbf{h} 1}}(y)\right)
$$

where the union is over all $\bar{e}_{\mathbf{h l}}$, such that $\ell\left(w \cdot \gamma_{\mathbf{h l}}\right)=\ell(w)-\sum_{c=1}^{m}\left(n_{l_{c}+1}-n_{h_{c}}\right)$, and for each $\bar{e}_{\mathbf{h l}}$ as above the preimage $\phi_{\bar{e}_{\mathbf{h l}}}^{-1}(Z)$ is given by the intersection (6.12), with $\bar{e}$ replaced by $\bar{e}_{\mathbf{h l}}$.

At this point we need the following

Lemma 6.5. The intersection

$$
\psi_{\bar{e}_{\mathbf{h} \mathbf{l}}}(y)^{-1}\left(\Omega_{\tilde{\alpha}_{i, j}^{\bar{e}_{\mathbf{h} 1}}}\right) \bigcap \psi_{\bar{e}_{\mathbf{h} \mathbf{l}}}(y)^{-1}\left(\Omega_{\tilde{w}^{\bar{e}_{\mathbf{h} 1}}}\right) \bigcap \pi^{-1}\left(\{y\} \times \bar{\Omega}_{v}(t)\right)
$$

is empty whenever $\bar{d} \neq \bar{e}_{\mathbf{h l}}$.

Granting this for a moment, let's complete the proof of Quantum Pieri.

Recall that $\mathcal{U}_{\bar{e}_{\mathrm{hl}}}(y)$ can be realized as a succesion of Grassmann bundles over an open subscheme $\mathcal{V} \subset\{y\} \times \mathcal{H} \mathcal{Q}_{\bar{d}-\bar{e}_{\mathrm{hl}}}$ (cf. the proof of Lemma 5.1). The above claim says that $Z$ is empty, except possibly when $\bar{d}$ is one of the multiindices $\bar{e}_{\mathbf{h l}}$ described above. In this case,

$$
\{y\} \times \mathcal{H} \mathcal{Q}_{\bar{d}-\bar{e}_{\mathbf{h l}}}=\mathcal{V}=\{y\} \times H_{\bar{d}-\bar{e}_{\mathbf{h l}}}=\{y\} \times F
$$

and $\mathcal{U}_{\bar{e}_{\mathbf{h} 1}}(y)$ is projective. Moreover, the map $\phi_{\bar{e}_{\mathbf{h} 1}}(y): \mathcal{U}_{\bar{e}_{\mathbf{h} 1}}(y) \rightarrow \mathcal{H} \mathcal{Q}_{\bar{d}}$ is an embedding, by Theorem $5.2(\mathrm{ii})$. It follows that the degree of $[Z]$ in the Chow ring of $\mathcal{H} \mathcal{Q}_{\bar{d}}$ is given by

$$
\int_{\mathcal{U}_{\bar{e}_{\mathbf{h} \mathbf{1}}(y)}} \psi_{\bar{e}_{\mathbf{h} \mathbf{1}}}(y)^{*}\left[\Omega_{\tilde{\alpha}_{i, j}^{\bar{e}_{\mathbf{h}}}}\right] \cup \psi_{\bar{e}_{\mathbf{h} \mathbf{1}}}(y)^{*}\left[\Omega_{\tilde{w}^{\bar{e}_{\mathbf{h}} \mathbf{1}}}\right] \cup \pi^{*}\left[\Omega_{v}\right]
$$

By applying the classical Pieri formula (Theorem 1.5) on $\tilde{F}_{\bar{e}_{\mathbf{h} 1}}$, we can rewrite $(6.13)$ as

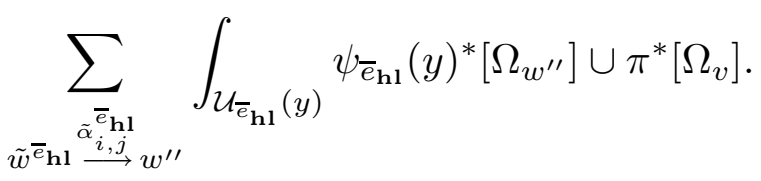
by

The subscheme $\psi_{\bar{e}_{\mathbf{h} 1}}(y)^{-1}\left(\Omega_{w^{\prime \prime}}\right)$ is the degeneracy locus inside $\mathcal{U}_{\bar{e}_{\mathbf{h} \mathbf{l}}}(y)$ determined

$$
\operatorname{rank}\left(V_{p} \otimes \mathcal{O} \rightarrow K_{q}^{*}\right) \leq r_{w^{\prime \prime}}(q, p), p=1, \ldots, n, q \in\left\{m_{1}, \ldots, m_{a}\right\}
$$

By Kleiman's transversality theorem, we may assume that both $\psi_{\bar{e}_{\mathbf{h l}}}(y)^{-1}\left(\Omega_{w^{\prime \prime}}\right)$ and the intersection $\psi_{\bar{e}_{\mathbf{h l}}}(y)^{-1}\left(\Omega_{w^{\prime \prime}}\right) \bigcap \pi^{-1}\left(\Omega_{v}\right)$ have the expected codimension. Hence 
in the Chow ring of $\mathcal{U}_{\bar{e}_{\mathbf{h} 1}}(y)$. Recall that $\pi: \mathcal{U}_{\bar{e}_{\mathbf{h} 1}}(y) \rightarrow F$ can be realized as a succesion of Grassmann bundle projections (cf. the proof of Lemma 5.1). By applying Lemma $6.4(i)$ to each of these Grassmann bundles, starting from the top, we get that the image of $\psi_{\bar{e}_{\mathbf{h l}}}(y)^{-1}\left(\Omega_{w^{\prime \prime}}\right)$ under the projection $\pi$ is the Schubert variety $\Omega_{w^{\prime \prime \prime}} \subset F$, where $w^{\prime \prime \prime}$ is the permutation (in $S$ !) obtained from $w^{\prime \prime}$ by the succesive applications of Lemma $6.4(\mathrm{i})$. By Lemma $6.4(\mathrm{ii})$, it follows that $\pi_{*}\left[\psi_{\bar{e}_{\mathbf{h l}}}(y)^{-1}\left(\Omega_{w^{\prime \prime}}\right)\right]=0$, unless the condition (6.8) is satisfied in every instance where we have used Lemma $6.4(i)$, in which case $\pi_{*}\left[\psi_{\bar{e}_{\mathbf{h l}}}(y)^{-1}\left(\Omega_{w^{\prime \prime}}\right)\right]=\left[\Omega_{w^{\prime \prime \prime}}\right]$.

Moreover, if this happens, the permutation $w^{\prime \prime \prime}$ is obtained from $w^{\prime \prime}$ by applying succesively the receipe (6.9). Using the fact that the simple transpositions $s_{i}$ and $s_{j}$ commute whenever $i$ and $j$ are not consecutive integers, it follows easily that $w^{\prime \prime \prime}=w^{\prime \prime} \cdot \delta_{\mathbf{h l}}$ and

$$
\ell\left(w^{\prime \prime \prime}\right)=\ell\left(w^{\prime \prime} \cdot \delta_{\mathbf{h l}}\right)=\ell\left(w^{\prime \prime}\right)-m-\sum_{c=1}^{m}\left(n_{l_{c}}-n_{h_{c}-1}\right) .
$$

From the projection formula

$$
\int_{F} \pi_{*}\left[\psi_{\bar{e}_{\mathbf{h} \mathbf{l}}}(y)^{-1}\left(\Omega_{w^{\prime \prime}}\right) \bigcap \pi^{-1}\left(\Omega_{v}\right)\right]=\int_{F}\left[\Omega_{w^{\prime \prime} \cdot \delta_{\mathbf{h} 1}}\right] \cup\left[\Omega_{v}\right] .
$$

By Theorem 1.2 the latter intersection number vanishes, unless $v$ is the permutation in $S$ dual to $w^{\prime \prime} \cdot \delta_{\mathbf{h l}}$, in which case it is equal to 1 . This implies that the same holds for the intersection number (6.13).

Summarizing, $\operatorname{deg}[Z]$ vanishes, unless all the conditions stated in Theorem 3.1' are satisfied, in which case it is equal to 1 , and moreover, we have seen that $\operatorname{deg}[Z]=$ $\left\langle\Omega_{\alpha_{i, j}}, \Omega_{w}, \Omega_{v}\right\rangle_{\bar{d}}$. This completes the proof of Quantum Pieri.

Proof of Lemma 6.5. For simplicity, we will omit $\mathbf{h}$ and $\mathbf{l}$ from the notation. We recall first the situation we're dealing with. There is a diagram

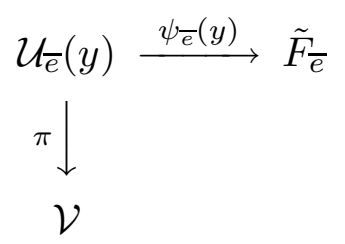

with $\{y\} \times H_{\bar{d}-\bar{e}} \subset \mathcal{V} \subset\{y\} \times \mathcal{H} \mathcal{Q}_{\bar{d}-\bar{e}}$ and $\pi$ a composition of Grassmann bundle projections. Let

$$
W:=\tilde{\Omega}_{\alpha_{i, j}}^{\bar{e}}(y) \bigcap \tilde{\Omega}_{w}^{\bar{e}}(y)=\psi_{\bar{e}}(y)^{-1}\left(\Omega_{\tilde{\alpha}_{i, j}^{\bar{e}}}\right) \bigcap \psi_{\bar{e}}(y)^{-1}\left(\Omega_{\tilde{w}^{\bar{e}}}\right)
$$

We may assume that $W$ is irreducible, of the expected codimension $\ell\left(\tilde{\alpha}_{i, j}^{\bar{e}}\right)+\ell\left(\tilde{w}^{\bar{e}}\right)$, while the intersection $W \bigcap \pi^{-1}\left(\{y\} \times \bar{\Omega}_{v}(t)\right)$ is a nonempty finite set consisting of reduced points, and supported on $\pi^{-1}\left(\{y\} \times H_{\bar{d}-\bar{e}}\right)$. It follows then that $\pi(W) \bigcap(\{y\} \times$ $\left.\Omega_{v}(t)\right)$ is a nonempty zero-dimensional subscheme of $\{y\} \times H_{\bar{d}-\bar{e}}$. By Lemma 6.1 , this would imply $\bar{d}=\bar{e}$, and therefore conclude the proof, if we can show that $\pi(W) \bigcap\left(\{y\} \times H_{\bar{d}-\bar{e}}\right)$ is of the form $Y(y)$, for some subvariety $Y \subset F$. Set $Y:=e v_{y}(\pi(W))$, where $e v_{y}$ is the restriction of the evaluation map to $\{y\} \times H_{\bar{d}-\bar{e}}$. 
exists a map $f: \mathbb{P}^{1} \rightarrow F$ with $[f] \in \pi(W)$, then for every $g: \mathbb{P}^{1} \rightarrow F$ such that $g(y)=f(y)$ we have $[g] \in \pi(W)$ as well. The map $f$ is represented by a sequence of subbundles

$$
S_{1} \subset S_{2} \subset \cdots \subset S_{k} \subset V^{*} \otimes \mathcal{O}_{\mathbb{P}^{1}}
$$

By assumption, there exists a point in $W \subset \mathcal{U}_{\bar{e}}(y)$, lying over $[f]$. This is equivalent to saying that for every $i \in\{1, \ldots k\}$ there exist quotients

$$
S_{i}(y) \rightarrow \mathbb{C}^{e_{i}}
$$

of the fibres at $y$, together with compatible maps $\mathbb{C}^{e_{i}} \rightarrow \mathbb{C}^{e_{i+1}}$, and which satisfy the degeneracy conditions defining $\tilde{\Omega}_{\alpha_{i, j}}^{\bar{e}}(y)$ and $\tilde{\Omega}_{w}^{\bar{e}}(y)$. If $g$ is another map and $g(y)=f(y)$, then the flag of fibres at $y$ for the sequence of subbundles corresponding to $g$ coincides with

$$
S_{1}(y) \subset S_{2}(y) \subset \cdots \subset S_{k}(y) \subset V^{*} \otimes \mathcal{O}_{y}
$$

Hence we can take the same quotients $(*)$ to obtain a point in $W$ lying over $[g]$, and the Lemma is proved.

\subsection{Proof of Orthogonality.}

Lemma 6.6. Let $v_{1}, \ldots, v_{N} \in S$ and $w_{1}, \ldots w_{M} \in S$ be two collections of permutations satisfying the conditions

(i) each $v_{m}$ (respectively, $\left.w_{m}\right), 1 \leq m \leq N$ (respectively, $1 \leq m \leq M$ ) is a cycle $\alpha_{i, j}$, for some $i$ and $j$;

(ii) for each $j$, the number of cycles $\alpha_{i, j}$ among the $v_{m}$ 's (respectively, $w_{m}$ 's) is at most $n_{j+1}-n_{j}$;

(iii) $\sum_{i=1}^{N} \ell\left(v_{i}\right)+\sum_{j=1}^{M} \ell\left(w_{j}\right)>\ell\left(w^{\circ}\right)=\operatorname{dim} F$.

Then $\left\langle\Omega_{v_{1}}, \ldots, \Omega_{v_{N}}, \Omega_{w_{1}}, \ldots, \Omega_{w_{M}}\right\rangle_{\bar{d}}=0$, for every $\bar{d}$.

Proof. The condition ( iii) gives the result for $\bar{d}=(0, \ldots, 0)$, hence we may assume that $\bar{d}$ is not identically 0 , and that $\sum_{i=1}^{N} \ell\left(v_{i}\right)+\sum_{j=1}^{M} \ell\left(w_{j}\right)=\operatorname{dim} H_{\bar{d}}$. Let $y, t_{1}, \ldots, t_{M} \in \mathbb{P}^{1}$ be distinct points and let $\Omega_{v_{1}} \cap \cdots \bigcap \Omega_{v_{M}}:=Y \subset F$. By Lemma 5.7 , and the conditions $(i)$ and $(i i)$, for every multiindex $\bar{e}$ we have the inequality

$$
\sum_{m=1}^{N}\left(\ell\left(v_{m}\right)-\ell\left(\tilde{v}_{m}^{\bar{e}}\right)\right) \leq \sum_{j=1}^{k} e_{j}\left(n_{j+1}-n_{j}\right) .
$$

Using this, the same argument as in the proofs of Theorem 4.4 (ii) and Lemma 6.5 shows that the intersection

$$
\bar{\Omega}_{v_{1}}(y) \bigcap \cdots \bigcap \bar{\Omega}_{v_{N}}(y) \bigcap \bar{\Omega}_{w_{1}}\left(t_{1}\right) \bigcap \cdots \bigcap \bar{\Omega}_{w_{M}}\left(t_{M}\right)
$$

misses the boundary of $\mathcal{H} \mathcal{Q}_{\bar{d}}$. Therefore we can apply Lemma 6.2 to conclude that

$$
\left\langle\Omega_{v_{1}}, \ldots, \Omega_{v_{N}}, \Omega_{w_{1}}, \ldots, \Omega_{w_{M}}\right\rangle_{\bar{d}}=\int[\overline{Y(y)}] \cup\left[\bar{\Omega}_{w_{1}}\left(t_{1}\right)\right] \cup \cdots \cup\left[\bar{\Omega}_{w_{N}}\left(t_{N}\right)\right]
$$


Now the same reasoning can be applied to the collection $w_{1}, \ldots, w_{M}$ to reduce the integral in (6.16) to one involving only two subvarieties of $F$. By Lemma 6.1, all such intersection numbers vanish whenever $\bar{d} \neq(0, \ldots, 0)$.

Proof of Theorem 3.16. It follows at once from the orthogonality of the classical Giambelli polynomials and the definition of $P_{w}^{q}$ that it suffices to consider the case $\ell(w)+\ell(v)>\ell\left(w^{\circ}\right)$. This in turn follows if we show that

$$
\left\langle\left\langle G_{\Lambda_{1} \Lambda_{2} \ldots \Lambda_{k}} G_{\Psi_{1} \Psi_{2} \ldots \Psi_{k}}\right\rangle\right\rangle=0
$$

for all partitions $\Lambda_{1}, \ldots, \Lambda_{k}, \Psi_{1}, \ldots, \Psi_{k}$ such that

$$
\left|\Lambda_{1}\right|+\cdots+\left|\Lambda_{k}\right|+\left|\Psi_{1}\right|+\cdots+\left|\Psi_{k}\right|>\ell\left(w^{\circ}\right) .
$$

Recall that $G_{\Lambda_{1} \Lambda_{2} \ldots \Lambda_{k}}$ was defined as the product $G_{\Lambda_{1}}^{(1)} G_{\Lambda_{2}}^{(2)} \ldots G_{\Lambda_{k}}^{(k)}$, while each $G_{\Lambda_{j}}^{(j)}$ is itself a product of at most $n_{j+1}-n_{j}$ factors of type $G_{i}^{j}$, for various $i$ 's (and the same for $G_{\Psi_{1} \Psi_{2} \ldots \Psi_{k}}$ ). But we already know by the special case of the Quantum Giambelli formula (see Theorem $3.9(i)$ ) that for every $i$ and $j$ the polynomial $G_{i}^{j}$ represents the Schubert class $\left[\Omega_{\alpha_{i, j}}\right]$ in $Q H^{*}(F)$ ! Therefore the product $G_{\Lambda_{1} \Lambda_{2} \ldots \Lambda_{k}} G_{\Psi_{1} \Psi_{2} \ldots \Psi_{k}}$ coincides with the quantum product obtained by replacing each $G_{i}^{j}$ by the corresponding $\left[\Omega_{\alpha_{i, j}}\right]$. By Remark 4.8, the relation (6.17) is a consequence of Lemma 6.6.

\section{REFERENCES}

[AS] A. Astashkevich and V. Sadov, Quantum cohomology of partial flag manifolds $F_{n_{1}, \ldots, n_{k}}$, Comm. Math. Phys. 170 (1995), 503-528.

[Beh] K. Behrend, Gromov-Witten invariants in algebraic geometry, preprint (1996).

[BF] K. Behrend and B. Fantechi, The intrinsic normal cone, preprint (1996).

[BM] K. Behrend and Y. Manin, Stacks of stable maps and Gromov-Witten invariants, preprint (1995).

[BGG] I. N. Bernstein, I. M. Gelfand, and S. I. Gelfand, Schubert cells and cohomology of the space $G / P$, Russian Math. Surveys 28 (1973), 1-26.

[Be] A. Bertram, Quantum Schubert calculus, Adv. Math. (to appear).

[Bor] A. Borel, Sur la cohomologie des espaces fibrés principaux et des espaces homogènes des groupes de Lie compacte, Ann. of Math. (2) 57 (1953), 115-207.

[C-F1] I. Ciocan-Fontanine, Quantum cohomology of flag varieties, Internat. Math. Res. Notices, no. 6 (1995), 263-277.

[C-F2] - The quantum cohomology ring of flag varieties, preprint (1996).

[D] M. Demazure, Désingularization des variétés de Schubert généralisée, Ann. Scient. École Normale Sup. 7 (1974), 53-88.

[E] C. Ehresmann, Sur la topologie des certaines espaces homogènes, Ann. of Math. 35 (1934), 396-443.

[FGP] S. Fomin, S. Gelfand, and A. Postnikov, Quantum Schubert polynomials, preprint (1996).

[F1] W. Fulton, Flags, Schubert polynomials, degeneracy loci and determinantal formulas, Duke Math. Journal 65 (1991), 381-420.

[F2] Intersection Theory, Springer Verlag, 1984.

[FP] W. Fulton and R. Pandharipande, Notes on stable maps and quantum cohomology, Institut Mittag-Leffler Report No. 4 (1996/97).

[GK] A. Givental and B. Kim, Quantum cohomology of flag manifolds and Toda lattices, Comm. Math. Phys. 168 (1995), 609-641.

[Kim1] B.Kim, Quantum cohomology of partial flag manifolds and a residue formula for their intersection pairing, Internat. Math. Res. Notices, no. 1 (1995), 1-16.

[Kim2] — On equivariant quantum cohomology, Internat. Math. Res. Notices, no. 17 (1996), 
[Kim3] _ Gromov-Witten invariants for flag manifolds, UC Berkeley Thesis (1996).

[KiMa] A.N. Kirillov and T. Maeno, Quantum double Schubert polynomials, quantum Schubert polynomials and Vafa-Intriligator formula, preprint (1996).

[Kl] S. Kleiman, The transversality of a general translate, Compositio Math. 38 (1974), 287297.

[Kon] M. Kontsevich, Enumeration of rational curves via torus actions, in The moduli space of curves, R. Dijkgraaf, C. Faber and G. van der Geer, eds., Birkhauser, 1995, pp. 335-368.

[KM] M. Kontsevich and Y. Manin, Gromov-Witten classes, quantum cohomology and enumerative geometry, Comm. Math. Phys. 164 (1994), 525-562.

[LS1] A. Lascoux and M.-P. Schützenberger, Polynômes de Schubert, C.R. Acad. Sci. Paris 294 (1982), 447-450.

[LS2] - Symmetry and flag manifolds, in Invariant Theory, F. Gherardelli ed., Lecture Notes in Math., vol. 996, Springer, Berlin, 1983, pp. 118-144.

[Lau] G. Laumon, Un anlogue global du cône nilpotent, Duke Math. Journal 57 (1988), 647-671.

[LT1] J. Li and G. Tian, The quantum cohomology of homogeneus varieties, J. Algebraic Geom. (to appear).

[LT2] - Virtual moduli cycles and Gromov-Witten invariants, preprint (1996).

[M] I. G. Macdonald, Notes on Schubert Polynomials, LCIM, Département de mathématiques et d'informatique, Université du Québec à Montréal, 1991.

[MS] D. McDuff and D. Salamon, J-holomorphic curves and quantum cohomology, Univ. Lecture Ser. 6, Amer. Math. Soc., Providence, RI, 1994.

[Pe] D. Petersen, unpublished (1996).

[RT] Y. Ruan, G. Tian, A mathematical theory of quantum cohomology, J. Differential Geom. 42 (1996), 259-367.

[ST] B. Siebert and G. Tian, On quantum cohomology of Fano manifolds and a formula of Vafa and Intriligator, preprint (1994).

[S] F. Sottile, Pieri's rule for flag manifolds and Schubert polynomials, Annales de L'Institut Fourier 46 (1996), 89-110.

[T] G. Tian, Quantum cohomology and its associativity, preprint (1995).

[V] C. Vafa, Topological mirrors and quantum rings, in Essays on Mirror Manifolds, S.T. Yau ed., International Press, Hong Kong, 1992.

[W] E. Witten, Topological sigma model, Commun. Math. Phys. 118 (1988), 411-449.

Institut Mittag-Leffler, Auravägen 17, S-182 62, Djursholm, Sweden

E-mail address: ciocan@ml.kva.se 